\title{
Geochemical and petrological characteristics of xenoliths in Mansehra Granite, NW Himalaya, Pakistan: implications for petrogenesis and tectonic settings
}

\author{
${ }^{1}$ Institute of Geochemistry, Chinese Academy of Sciences, Guiyang 550081, China; *Corresponding author, E-mail: tehseenabbas11@yahoo.com \\ ${ }^{2}$ Department of Geological Sciences, University of Texas at El Paso, El Paso, TX 79968, United States \\ ${ }^{3}$ Department of Earth and Environmental Sciences, Kagoshima University, Kagoshima, Japan \\ ${ }^{4}$ Institute of Geology, University of the Punjab, Lahore, 54590, Pakistan \\ ${ }^{5}$ National Centre of Excellence in Geology, University of Peshawar, Peshawar, 25130, Pakistan
}

(Received: April 17, 2019; Revised accepted: September 16, 2019)

https://doi.org/10.18814/epiiugs/2019/019022

Mansehra Granite (MG) is massive and sheared which contains light-gray to jet-black schistosed and light-gray micaceous quartzite restites. The later possess relatively higher quartz as compared with mica contents whereas the former has predominant quartz and feldspar. Enclosure of apatite and zircon in micas and absence of sillimanite in schistosed and non-foliated facies suggest that these rocks are restite in nature. Presence of abundant and larger mica flakes at contact of restites with $M G$ indicates exposure of these flakes at relatively higher temperature of granitic melt $\left(623.7-886.6^{\circ} \mathrm{C}\right)$ particularly at their margins. However, central part of restites remained unaffected due lesser impact of elevated temperature. Schistose and surmicaceous restites show crenulation cleavage and preserve structural antiquity during amalgamation in felsic melt. Geochemically, restites and Tanawal Formation indicate calc-alkaline to high $K$ calc-alkaline domain with orogenic syn-collisional settings. Geochemical characteristics and modal mineralogy of restites and Tanawal Formation are similar. This study suggests that former has been derived from the later and their field relationships and geochemical signatures provide credible evidence in favour of S-type trait of Mansehra Granite. In geochemical diagrams, $M G$ portrayed calc-alkaline, peraluminous S-type nature and its magma was generated from heterogeneous Tanawal Formation via fractional crystallization.

\section{Introduction}

The Mansehra Granitic Complex (MGC) is exposed in the Lesser Himalaya, NW of Pakistan (Fig. 1). The Mansehra Granite, a main member of the MGC, occurs as sub-concordant to discordant batho- lith which covers an area of $100 \mathrm{~km}^{2}$. The granite is generally massive but sheared at places due to ductile deformation (Shams, 1961). The Mansehra magmatic body is calc-alkaline and peraluminous having S-type traits formed through the anatexis of the Precambrian Tanawal Formation (Jan et al., 1981). The granite is intruded by basic dykes (Shams, 1967a). It is a binary mica and porphyritic granite which has K-feldspar phenocrysts. Apart from phenocrysts, the Mansehra Granite also contains light-gray to dark-gray as well as jet black xenoliths of varying size which may represent rock fragments entrapped during emplacement of magma or remnants of un-melted protolith. In particular, xenoliths are foreign rock fragments found in rocks having diverse composition from the enveloping rock which may indicate enclaves or restites (Vernon, 1983; Bacon, 1986). Study of xenoliths in granites provides valuable perception about magmatic evolution that may deliver credible evidence about petrogenetic processes (Didier and Barbarin, 1991; Waight et al., 2001). Although the derivation of xenoliths is still an issue of debate, however, it has been acknowledged that the xenoliths convey an exclusive opportunity to constrain the petrogenesis and provide valuable facts regarding the tectonic settings that prevailed during their genesis (Sarjoughian et al., 2017).

Granitic rocks of the Mansehra area has been investigated by many workers (e.g, Calkins and Matin, 1968; Honeggar et al., 1982; Chaudhry et al., 1989; Ashraf, 1992). Earlier workers have presented general description of the rocks of the area. However, Shams (1961) carried out first systematic study on the granitic and associated metasedimentary rocks of the Mansehra area. Later, Shams (1971) undertaken detailed geological work and presented classification of rocks of the Mansehra. Naeem et al. (2016) carried out geochronological study of the Mansehra Granite and presented age of $516 \pm 16 \mathrm{Ma}$ based on $\mathrm{Rb} /$ $\mathrm{Sr}$ method. The Mansehra Granite contains xenoliths of varying size and composition that necessitates to investigate their nature and mode of occurrence which has a significant bearing on the petrological aspects of the MGC magmatic rocks. Hence, there is a dire need to carry out a thorough investigation of these xenoliths. Field relation- 


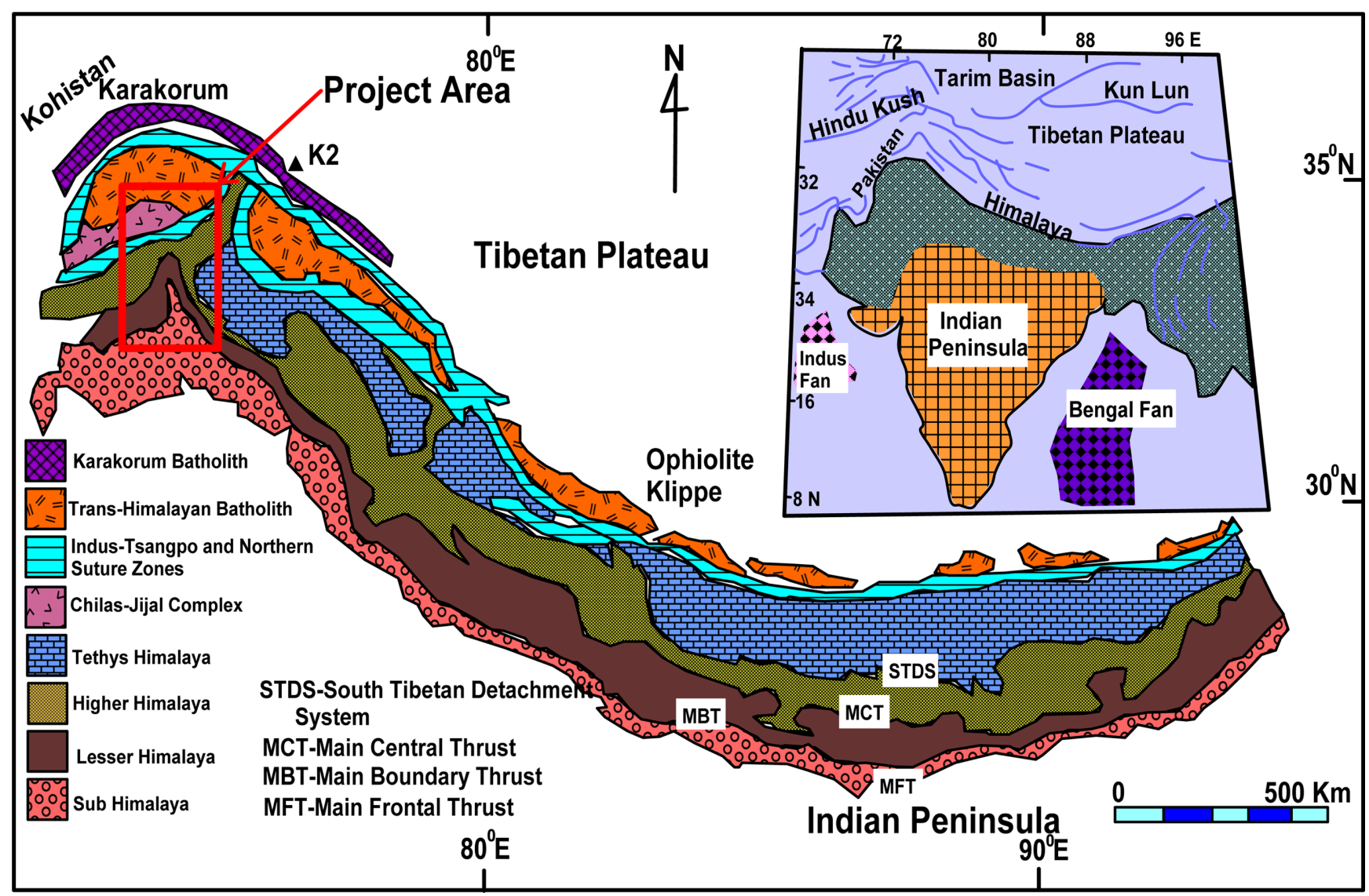

Figure 1. Map shows location of study area and main tectonic sub-division of Himalaya (modified after Ganser, 1964; Sorkhabi and Arita, 1997; Paudel and Arita, 2000).

ships augmented with petrography and geochemistry of these xenoliths integrated with previous findings of the MGC rocks will furnish the credible evidence regarding petrogenesis of the xenoliths and Mansehra Granite. In the present research, we aim to provide new geochemical data and deliver insights into: (1) petrogenesis and compositional characteristics of xenoliths and Mansehra pluton (2) their tectonic implications.

\section{Geology of the Area}

The Himalayan mountain range, resulted from collision between the Indian and Eurasian plates, has been sub-divided into four major tectonic elements from north to south including Sub-Himalaya, Lesser Himalaya, Higher and Tethys Himalaya (Ganser, 1964; Le Fort, 1975; Hodges, 2000). The Lesser Himalaya is demarcated by the Main Central Thrust (MCT) in the north while delineated by Main Boundary Thrust (MBT) in the south. In Mansehra area, the Lesser Himalaya consists of igneous and metamorphic rocks constituting the Mansehra Granitic Complex (MGC) which is surrounded by metasediments of the Tanawal Formation, comprised of pelite, psammite, phyllite, quartzite and schists (Fig. 2). The MGC is principally composed of several plutonic bodies including medium to coarse-grained, binary mica, porphyritic and massive to sheared Mansehra Granite and poorly porphyritic to non-porphyritic tourmaline bearing massive Hakale Granite.
In addition, fine-grained microgranites, massive to sheared leucogranites and associated tabular as well as pod-like pegmatitic and aplitic bodies are also exposed (Ashraf and Chaudhry, 1976a, b; Naeem et al., 2016). Mansehra Granite is the main member of this complex which contains variety of xenoliths. Field description of xenoliths and enclosing Mansehra Granite is given below.

Along the Susalgali-Khaki (S-K) traverse, the sheared Mansehra Granite has microcline phenocrysts with groundmass. The sheared granite contains 1 to $5 \% \mathrm{~K}$-feldspar phenocrysts having length ranging from 0.5 to $3 \mathrm{~cm}$ (Fig. 3a). Lenticular to elongated xenoliths found in the granite have light-gray to jet-black rusty colour (Fig. 3b-c). Size of these xenoliths varies between $2.5 \times 2$ and $12 \times 10 \mathrm{~cm}$. Light-grey oval xenoliths reveal abundant K-feldspar phenocrysts with parallel alignment (Fig. 3d). In contrast, elongated and sub-rounded xenoliths contain hematite and show reddish-brown colouration due to oxidation of iron around their margins (Fig. 3e). Lenticular xenoliths are fractured at places. Mineralogically, xenoliths contain quartz, feldspar, biotite, muscovite and rusty brown hematite. These xenoliths represent diffused, irregular and sharp contacts with the granitoid host.

Along the Oghi-Darband traverse (western part of the granite, Fig. 2), the Mansehra Granite is massive having microcline phenocrysts with weak flow-foliation. Scarce microcline crystals exhibit augen structure. Alignment of tadpole-shaped phenocrysts is also perceived at number of places. The granite comprises $1-15 \% \mathrm{~K}$-feldspar phenocrysts from $0.6 \mathrm{~mm}$ to $7 \mathrm{~cm}$ in size. The granite contains light-gray to jet-black, 


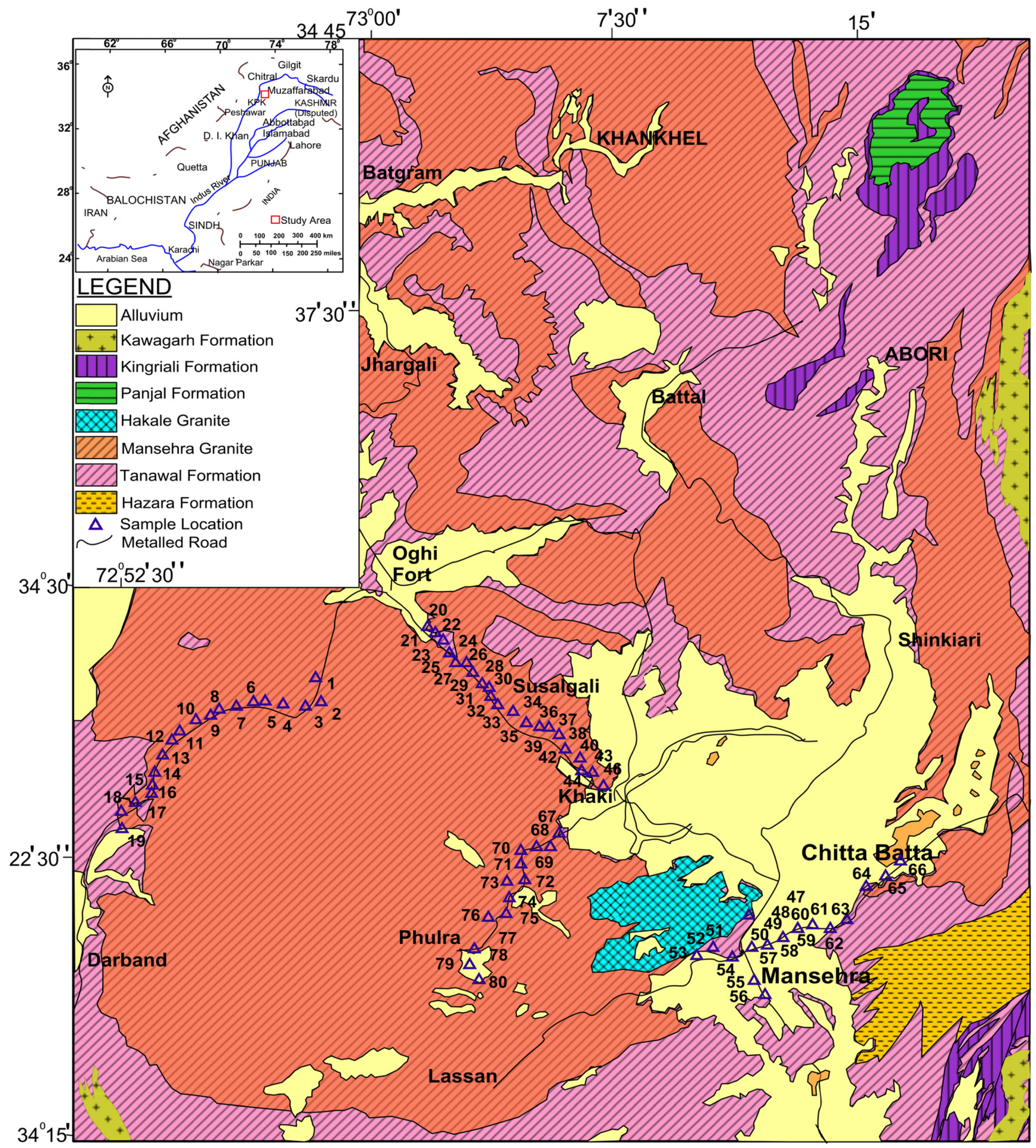

Figure 2. Geological map of Mansehra area showing samples location (modified after Calkins et al., 1975).

rounded to angular as well as lenticular xenoliths (Fig. 3f-g). K-feldspar crystals in the granite are aligned parallel to the margins of lightgray and jet-black xenoliths. Sizes of the xenoliths range from $3 \times 2.8$ to $12 \times 8 \mathrm{~cm}$ which contains quartz, feldspar, biotite and muscovite. These xenoliths reveal sharp, irregular and diffused contact with the host Mansehra Granite (Fig. 3g).

In Mansehra area, the granite is massive which contains K-feldspar phenocrysts along with xenoliths at the contact between the granite and Tanawal Formation. These xenoliths are light-gray to jet-black, rounded to oval in shape. Size of these xenoliths ranges from $4.5 \times 2$ to $15 \times 7.5 \mathrm{~cm}$. Jet black xenoliths are either devoid of or contain scarce (1-2\%) K-feldspar crystals aligned parallel around the rim of these xenoliths (Fig. 3h). At places, rim of these xenoliths depicts rusting due to oxidation of iron-bearing minerals. Light to dark-gray xenoliths encompasses $20 \% \mathrm{~K}$-feldspar crystals having $0.5 \times 0.3 \mathrm{~cm}$ size. Phenocrysts aligned around their border have size range $3-0.5 \mathrm{~cm}$. 

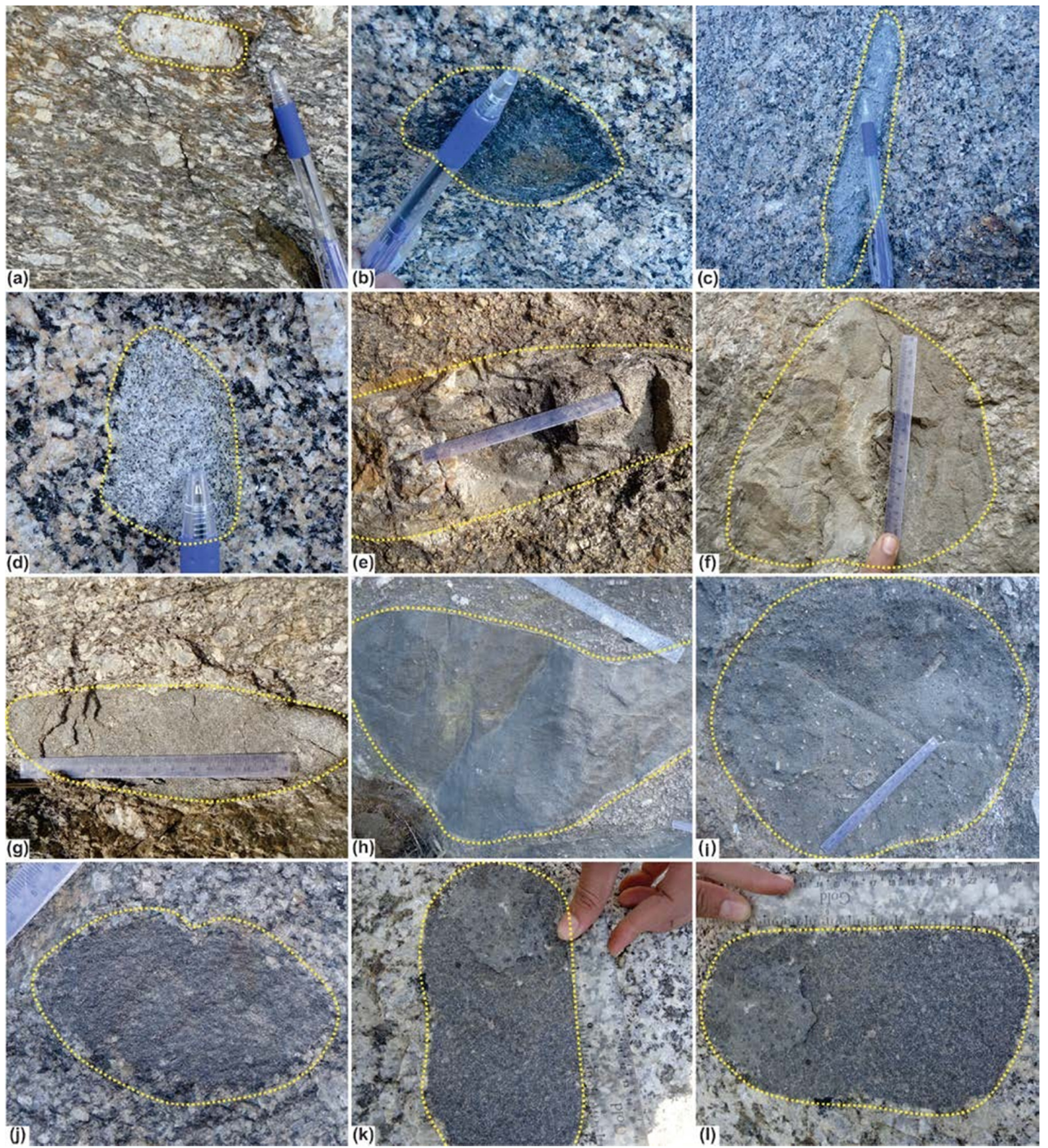

Figure 3. (a-k) represents field characteristics and features of xenoliths in Mansehra Granite exposed in diverse areas of NW Himalaya. (a) Sheared granite indicating deformed K-feldspar phenocrysts in Susalgali-Khaki area (b,c) Lenticular rusty jet-black and elongated inset bearing dark-gray xenolith in Mansehra Granite, Susalgali-Khaki area (d) Light-gray oval xenolith revealing parallel alignment of tadpole-shaped K-feldspar phenocrysts, Susalgali-Khaki area (e) Light-gray, elongated xenolith showing rusting at border and diffused contact with sheared Mansehra Granite, Susalgali-Khaki area (f,g) Light-gray, angular and lenticular xenoliths showing irregular contact with sheared Mansehra Granite, Susalgali-Khaki area (h) Minute K-feldspar phenocrysts around the rim of jet-black angular xenolith in massive Mansehra Granite, Mansehra area (i) K-feldspar insets bearing dark-gray rounded xenolith in massive Mansehra Granite, Mansehra area (j) Lenticular xenolith depicting diffused contact with host massive Mansehra Granite, Mansehra area (k) Jet-black xenolith in massive Mansehra Granite with diffused contact, Chitta-Batta area (l) Dark-gray, oval xenolith in massive Mansehra Granite, Phulra area. 
Dark-gray rounded xenoliths contain microcline crystals with $2 \times 1 \mathrm{~cm}$ size (Fig. 3i). Microcline phenocrysts with size $(2-5 \mathrm{~cm})$ are aligned along the margins of dark-gray, sub-rounded xenoliths. Apart from these, dark-gray xenoliths found in massive Mansehra Granite reveal schistosity. Mineralogically, xenoliths are comprised of quartz, feldspar, biotite, muscovite, K-feldspar phenocrysts and hematite. Xenoliths signify irregular and diffused contact with Mansehra Granite (Fig. 3j).

In Chitta-Batta, massive Mansehra Granite contains 3\% K-feldspar phenocrysts having maximum length of $2 \mathrm{~cm}$. The granite is massive, which contains dark-gray to jet-black, lenticular to oval xenoliths of $8 \times 4 \mathrm{~cm}$ size (Fig. $3 \mathrm{k}$ ). Field observation reveals that the xenoliths are composed of quartz, feldspar, biotite and muscovite. The xenoliths display irregular and diffused contact relations with granitoid host.

In Phulra area, Mansehra Granite is massive, medium-grained with weak to no flow-foliation. The granite contains angular to oval, darkgray to jet-black xenoliths (Fig. 31). Size of the xenoliths ranges from $4 \times 2$ to $13 \times 12.5 \mathrm{~cm}$. Megascopically, these varieties of xenoliths contain quartz, feldspar, biotite and muscovite and show irregular to diffused contact relationships with the Mansehra pluton (Fig. 31).

\section{Materials and Methods}

Fieldwork of the Mansehra area was carried, and representative xenoliths samples, enclosed in Mansehra Granite and a small number of samples from Tanawal Formation were collected from various localities (Fig. 2). In total, 81 samples were crumpled by jaw crusher at Pakistan Council of Scientific and Industrial Research Laboratories Complex (PCSIR), Lahore. Dust was removed from the surface of the fragments and kept at a temperature of $110^{\circ} \mathrm{C}$ to remove moisture content. Roll crusher was used to further reduce the size of these samples. The pulverized material was distributed over splitter to obtain representative fraction of each rock type. TEMA mill was used to get passing $200 \mathrm{~g}$ of 300 mesh powder that was stored in plastic bottles for chemical analysis of the samples. Moisture content of the samples was removed at $110^{\circ} \mathrm{C}$. For determination of loss on ignition, $5.0 \mathrm{~g}$ of every sample was taken in Platinum crucible and animated in furnace for 6-8 hours at $1050^{\circ} \mathrm{C}$. X-ray Fluorescence (XRF) method at Pakistan Atomic Energy Commission (PAEC) was used to determine major, minor and trace elements of the Mansehra Granite, xenoliths and Tanawal Formation. Fused lithium tetraborate discs were prepared to determine major and minor elements. Moisture-free $12 \mathrm{~g}$ powdered samples were mixed with lithium tetraborate in 1:5 proportion for the trace elements determination. Fusion machine was used to prepare pressed pellets, which analyses through X-ray Fluorescence spectrometer (model Pw 4400/24 Axios, PANalytical, Netherlands). Required corrections for matrix effect and the spectral overlapping were applied. In terms of RSD, the XRF data analytical accuracy was $\leq 2 \%$. The results of major, minor and trace element concentrations are presented in Tables 2 and 3.

\section{Petrography}

In total, 66 thin sections were prepared for petrography analysis of xenoliths, associated Mansehra Granite, and the Tanawal Formation. The comprehensive description is presented below.

\section{Light-gray Xenoliths}

Light-gray schistosed xenoliths found in massive granites of OghiDarband, Mansehra area, Chitta-Batta in addition to Phulra and sheared granites of Susalgali-Khaki area primarily consist of hypidiomorphic to allotriomorphic quartz and feldspar with subsidiary mica. The accessory phases include specifically zircon, apatite and magnetite. The rock is foliated, displays lepidoblastic texture (Fig. 4a) and asymmetrical crenulation cleavage occasionally. Most of the quartz grains are extremely strained. Few fractured quartz grains show sutured contacts. Muscovite and biotite flakes show preferred orientation and parallel to sub-parallel alignment (Fig. 4b). Biotite and muscovite show foliation, which contains apatite and zircon crystals (Fig. 4a, b). These schistosed xenoliths are augmented in micas, especially biotite and muscovite, therefore, can be considered as surmicaceous (Fig. 4a, b). Presence of surmicaceous enclaves in Mansehra Granite implies restite character of these xenoliths (White et al., 1991). Likewise, occurrence of zircon, apatite and absence of sillimanite suggest them restites rather than xenoliths (Pupin, 1980; Clemes and Wall, 1981; Pitcher, 1993; Fig. 4a, b). At places, biotite shows pinch and swell structure, indicating the effect of the strain. Subhedral to anhedral magnetite grains occur in dispersed form.

In comparison, light-gray micaceous quartzite xenoliths restricted in sheared Mansehra Granite (Susalgali-Khaki area), are mainly comprised of subhedral to anhedral quartz with subsidiary feldspar, biotite, muscovite and accessory zircon, apatite as well as magnetite. Quartz grains are strained and show strong undulatory extinction and sutured contacts (Fig. 4c). Quartz grains associated with broken muscovite and biotite flakes specify mylonization. Subhedral to anhedral plagioclase shows polysynthetic twinning and microcline depicts sericitization (Fig. 4c). Zircon and apatite are enclosed in biotite (Fig. 4d). Disseminated magnetite reveals presence of euhedral to subhedral crystals. Zircon and apatite found in micaceous quartzite indicates restite character of the xenoliths (Pupin, 1980; Pitcher, 1993; Fig. 4d). Meanwhile, absence of sillimanite and surmicaceous character of the rock also provides strong clue about restite nature of these xenoliths (Clemes and Wall, 1981; White et al., 1991; Fig. 4c, d). Modal mineralogical composition is revealed in Table 1.

\section{Dark-gray Xenoliths}

Massive and sheared Mansehra Granite contains dark-gray schistose xenoliths which principally comprised of subhedral to anhedral quartz and feldspar with subordinate biotite and muscovite. Zircon, hematite, apatite and magnetite occur as accessory minerals. At places, quartz grains are highly strained and have inclusions indicating sieve texture of the rock. Plagioclase and microcline occurs as subhedral to anhedral crystals. Biotite comprises of zircon surrounded by dark halos (Fig. 5a). Muscovite often indicates preferred alignment with parallel to sub-parallel pattern (Fig. 5b). Occurrence of zircon, surmicaceous nature and the absence of sillimanite in schistose rock specify them as restite (Pupin, 1980; Clemes and Wall, 1981; White et al., 1991; Pitcher, 1993; Fig. 5a, c). 

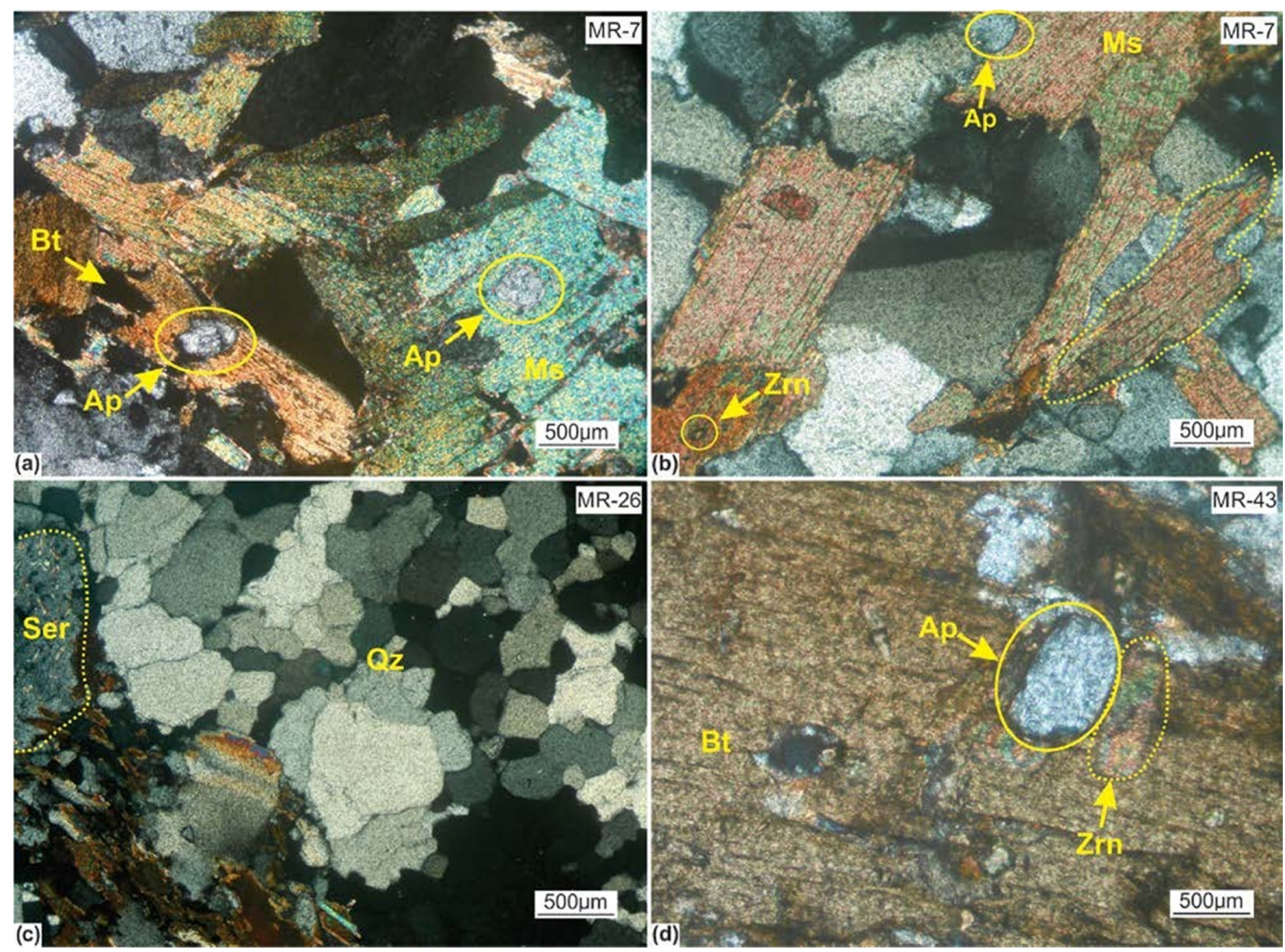

Figure 4. Microphotographs 4a-d depict various microscopic features of light gray restites: (a,b) Schistose restites sample MR-7 indicating schistosity, apatite (Ap) and zircon (Zrn) presence in biotite (Bt) and muscovite (Ms). (c) Micaceous quartzite restite MR-26 representing abundance of strained sutured quartz $(Q z)$ and presence of sericitization (Ser) in plagioclase. (d) Zircon and apatite occurrence in quartzite restite (MR-43).

The xenoliths show irregular and sharp contact with the enclosing Mansehra Granite manifested by marginal mylonization, abundance of sericitization, enrichment of deformed as well as larger biotite flakes, muscovite and development of myrmekites suggesting slow cooling of magma in compressional tectonic settings (Fig. 5d, e). At the contact of the xenoliths with the enclosing granite, mica shows bending and augen structure, which indicates the impact of deformation (Fig. $5 f)$. The rock is foliated, which reveals conspicuous lepidoblastic texture and interpreted with noticeable symmetrical to asymmetrical crenulation cleavage (Fig. 5g). In addition, hydrous mineral biotite was melted, re-crystallized and swirled and its core contains unmelted part at the contact, which can be typified as restite (Fig. 5h). Meanwhile, deformed biotite comprising zircon and associated with tinny quartz bands are stressed, which denotes tectonic impact (Fig. 5i). Modal mineral composition of the xenoliths is exhibited in Table 1.

\section{Mansehra Granite}

Enclosed Mansehra Granite is composed of feldspar, quartz and mica with accessory zircon, apatite and magnetite. Quartz grains occur in anhedral form, which are often strained and indicate undulose extinction (Fig. 5j). Muscovite occurs both in primary and secondary form in the granite. Coarse flakes indicate that muscovite is of primary nature, while minute flakes are indicative of secondary one in granite (Fig. 5j). So, textural characteristics discriminate primary and secondary muscovite (Saavedra, 1978; Zen, 1988). Subhedral to anhedral microcline indicates well-developed cross hatch twinning and comprises of stringlets however, fine to medium-grained, subhedral albite depicts polysynthetic twinning as well as sericitization (Fig. 5k). More importantly, quartz crystals coupled with deformed mica flakes designate process of mylonization (Fig. 5l). Pleochroic biotite occurs as arbitrarily oriented flakes having dark halos. Mica flakes are pinched and swelled occasionally which signifies tectonic foliation. Biotite commonly contains zircon and apatite inclusions (Xiang et al., 2006). Magnetite is infrequently present in euhedral form. In the meantime, tourmaline and hematite are identified sporadically. Petrographic studies reveal that the granite is augmented in Al-rich minerals, especially muscovite and indicates absence of allanite and titanite, which is suggestive of S-type character of this pluton (Saki, 2010). Modal mineralogical composition of the granite is displayed in Table 1 . 

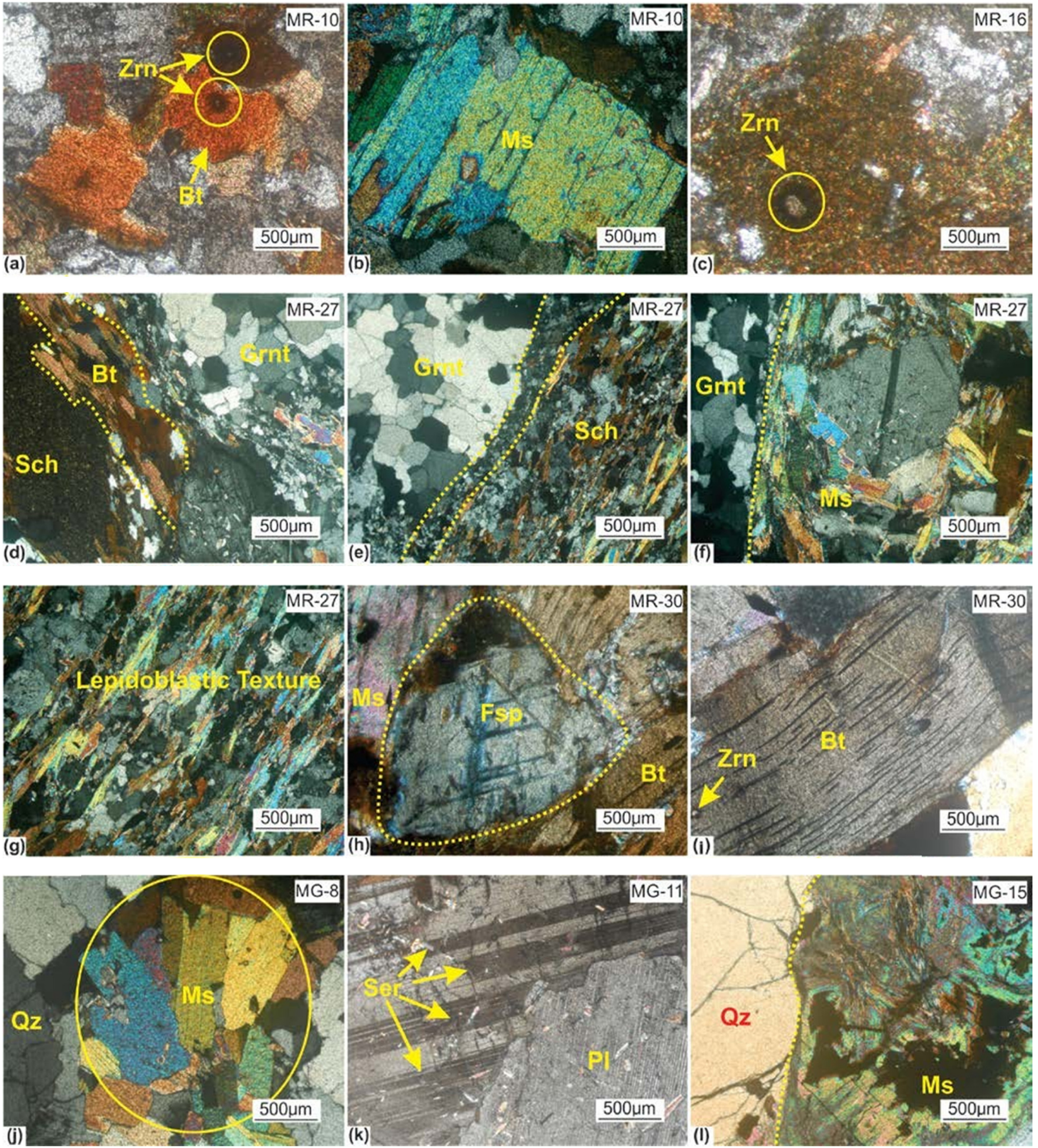

Figure 5. Microphotographs 5a-l portray diverse petrographic features of dark gray restites and Mansehra Granite: (a,b) schistose restite MR-10 signifies zircon (Zrn) halo in biotite (Bt) and presence of muscovite (Ms) sheets (c) Sample MR-16 specifies zircon (Zrn) with dark halo in schistose restite (d,e) Abundance of larger biotite (Bt) flake and marginal mylonization at the contact between schistose (Sch) restite and Granite (Grnt) respectively in sample MR-27 (f) Muscovite (Ms) flakes swirled around feldspar (Fsp) due to stress at the contact between schistose restite and Mansehra Granite (MR-27) (g) Lepidoblastic texture and noticeable crenulation cleavage in schistose restite, sample MR-27 (h) MR-30 indicates muscovite (Ms) and biotite (Bt) flake swirled around unmelted core of feldspar (Fsp) at the contact between schistose restite and Mansehra Granite (i) Tectonic bending in biotite (Bt) comprising zircon (Zrn) at the contact between schistose restite and Mansehra Granite in MR-30 (j,k) Mansehra Granite presenting strained quartz (Qz), primary (coarse) and secondary (smaller) muscovite (Ms) flakes and plagioclase (Pl) sericitization, twinning in MG-8,11 respectively (l) Deformed muscovite (Ms) and anhedral strained quartz (Qz) in Mansehra Granite MG-15. 
Table 1. Modal mineralogical composition (\%) of rocks from Oghi-Darband, Susalgali Khaki, Mansehra, Chitta-Batta and Phulra areas

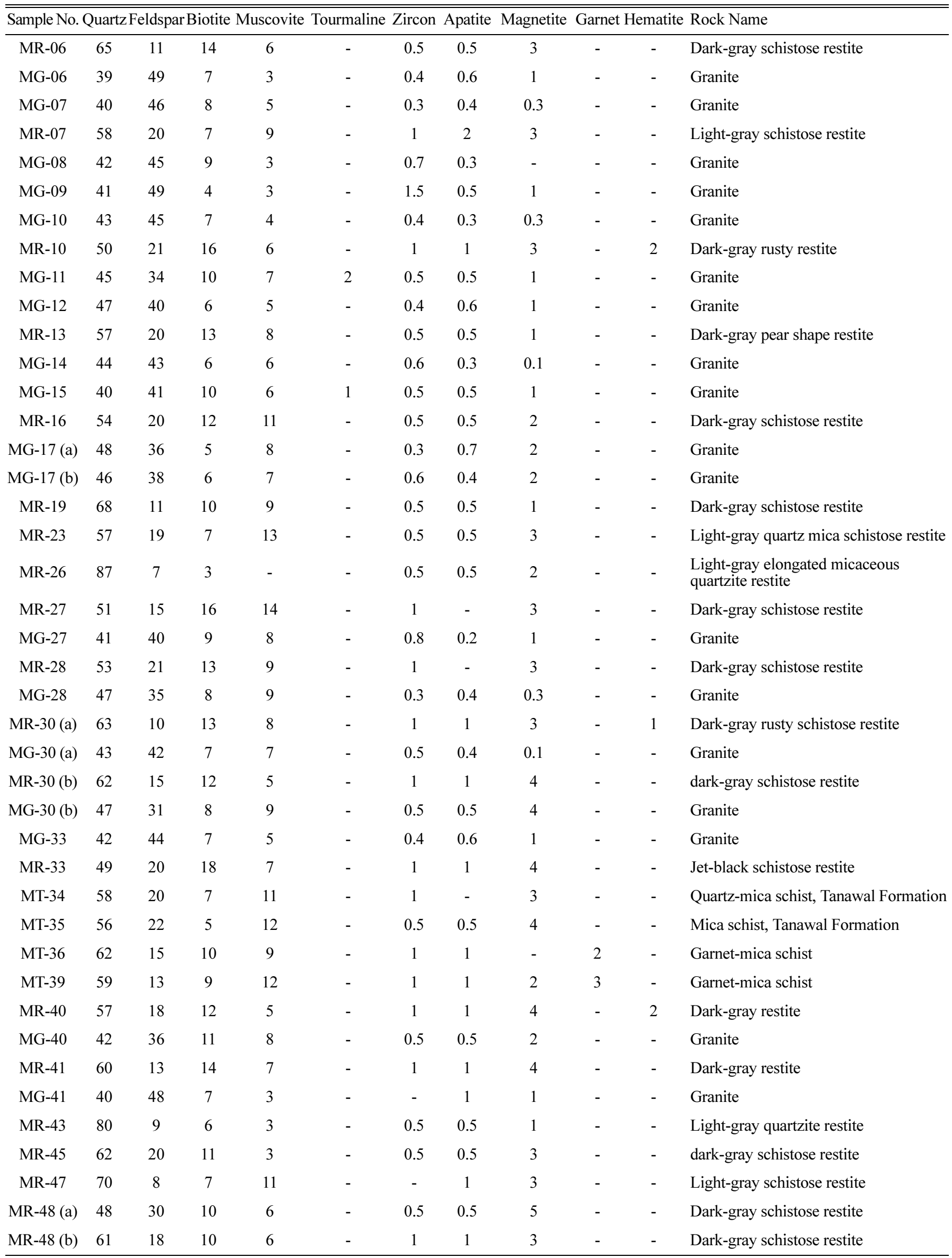


Table 1. Continued

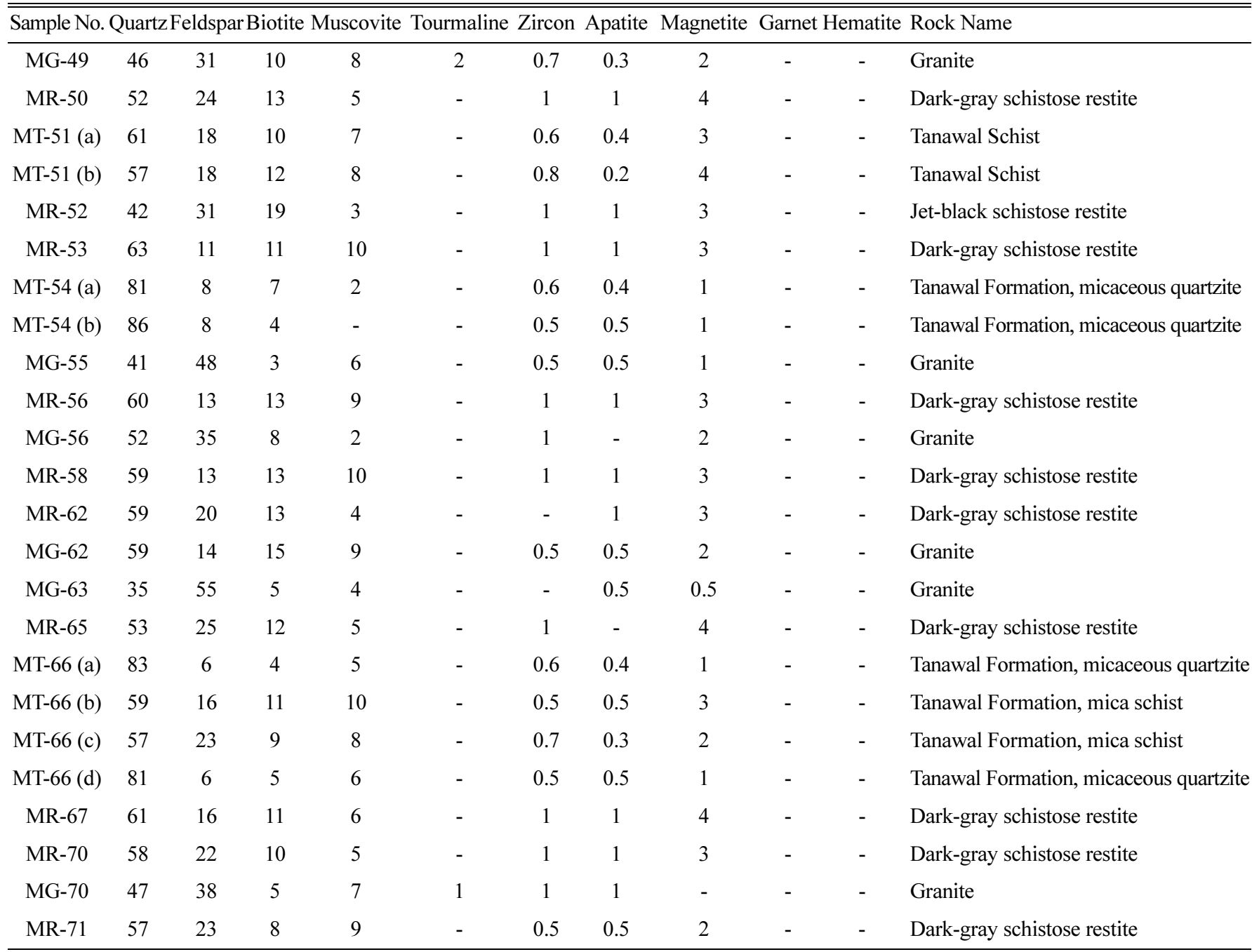

\section{Jet-black Xenoliths}

Jet-black xenoliths enclosed in Mansehra Granite are primarily comprised of hypidiomorphic to allotriomorphic quartz and feldspar with minor biotite, muscovite as well as accessory zircon, apatite and magnetite. The xenoliths depict lepidoblastic texture along with asymmetrical crenulation cleavage and foliation (Fig. 6a). Quartz grains reveal sutured contacts and are associated with mica at places (Fig. 6a). Anhedral feldspar shows sericitization. Meanwhile, magnetite occurs in distributed form having idiomorphic to allotriomorphic crystals and occasionally replaces muscovite (Fig. 6a). Moreover, mica reveals parallel to sub-parallel alignment with occurrence of euhedral zircon (Fig. 6b). It is worth stating that presence of euhedral zircon, lack of sillimanite and surmicaceous nature suggests the restite character of these xenoliths (Pupin, 1980; Clemes and Wall, 1981; White et al., 1991; Pitcher, 1993; Fig. 6b). Modal mineralogical composition of the jet-black xenoliths is revealed in Table 1.

\section{Tanawal Formation}

Schists of Tanawal Formation are predominantly comprised of sub- hedral to anhedral quartz and feldspar with subordinate mica and accessory apatite, magnetite and zircon. Textural characteristics reveal that the rocks are foliated and lepidoblastic (Fig. 7a). Quartz grains are patchily strained, interlocked and exhibit mosaic texture. Fine-grained muscovite substitutes quartz sporadically at number of places (Fig. 7a). Further, muscovite and biotite flakes reveal preferred orientation. Stressed muscovite symbolizes tectonic bending (Fig. 7a). Fine-grained quartz associated with broken mica suggests marginal mylonization (Fig. 7b). Meanwhile, mica associated with thin quartz bands are pinched and swelled, which characterizes tectonic foliation (Fig. 7b). Disseminated magnetite having euhedral to subhedral crystal shape replaces muscovite at number of places. Apart from these features, porphyroblasts of garnets are rarely found in the rocks (Fig. 7c). In contrast, micaceous quartzites of Tanawal Formation contain anhedral vastly strained quartz, feldspar with minor biotite, muscovite and accessory magnetite, zircon in addition to apatite. The rock is nonfoliated that exhibits granular texture. Biotite and muscovite flakes enclose zircon and apatite while magnetite occurs in disseminated form in these bodies. Mineralogical composition of samples of Tanawal Formation is expressed in Table 1. 

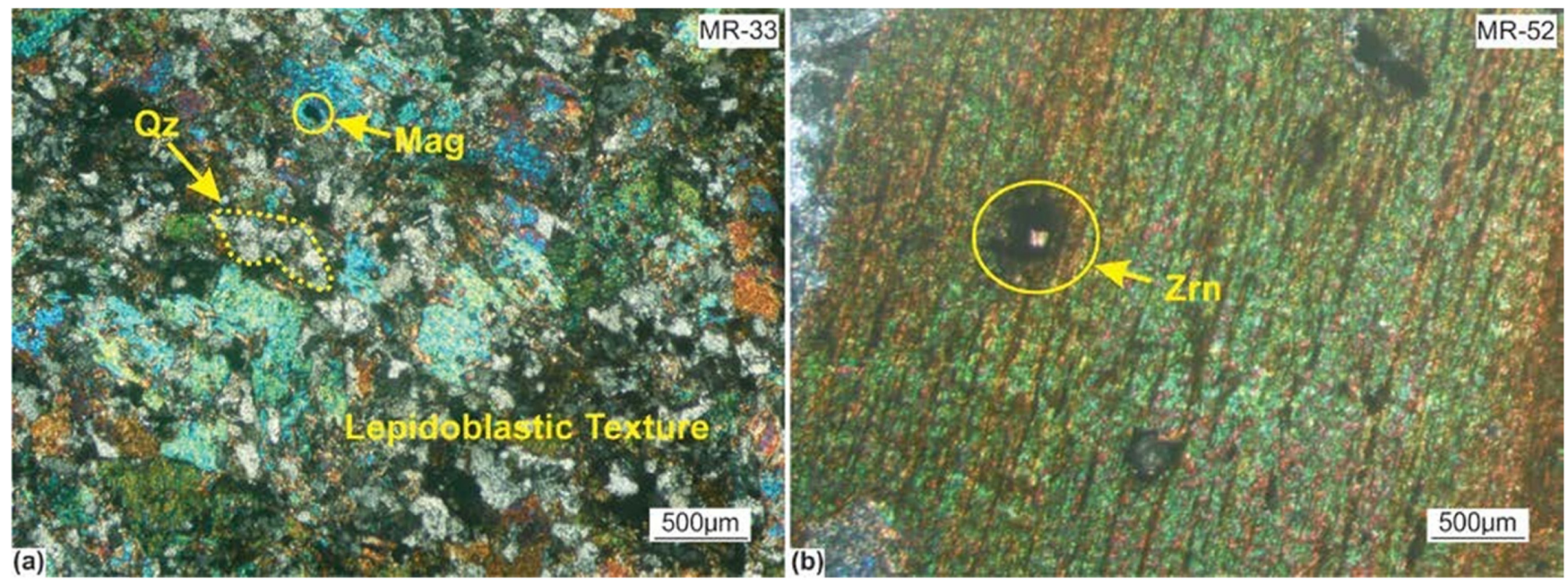

Figure 6. Microphotographs 6a-b portray lepidoblastic texture, magnetite (Mag) and euhedral zircon (Zrn) with dark halo in jet black schistose restite (MR-33, 52).
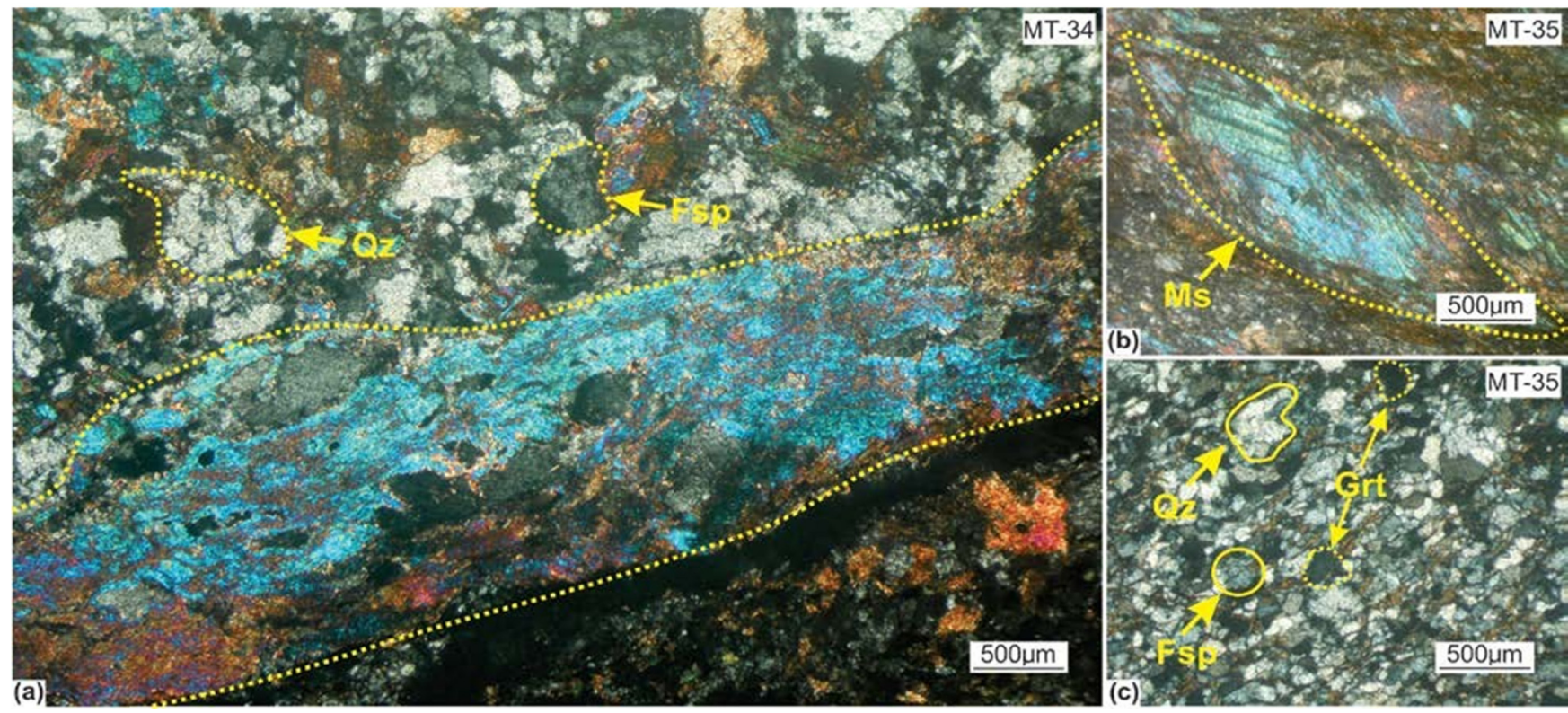

Figure 7. Microphotographs 7a-c signify microscopic features of Tanawal Formation in MT-34, 35 and 36: (a) tectonic bending in muscovite, quartz (Qz), feldspar (Fsp). (b) pinched and swelled muscovite (Ms) augen representing tectonic foliation. (c) garnet (Grt) presence in schist of Tanawal Formation.

\section{Analytical Results and Geochemistry}

Major, minor oxides and trace elements contents of eighty-one representative samples including xenoliths, Mansehra Granite and Tanawal Formation were determined through XRF. The results of various oxides (wt\%) and trace elements (ppm) contents of the analyzed samples are presented in Tables 2 and 3.

The geochemical data of Mansehra Granite as interpreted in $R_{1}-R_{2}$ and total alkali vs. $\mathrm{SiO}_{2}$ diagrams, principally placed $\mathrm{MG}$ in granite field (Fig. 8a, b). The chemical data of the xenoliths, Tanawal Formation and Mansehra Granite was plotted on geochemical classification diagrams. AFM diagram of the xenoliths and Tanawal Formation depicts calc-alkaline compositions except one sample, which indicates tholeiitic nature owing to relatively higher iron content (Fig. 9a). Nevertheless, all samples of MG exhibit calc-alkaline character in terms of AFM diagram (Fig. 9b). Likewise, $\mathrm{SiO}_{2}-\mathrm{K}_{2} \mathrm{O}$ plot placed the samples of xenoliths and Tanawal Formation in calc-alkaline, high $\mathrm{K}$ calc-alkaline and shoshonite series (Fig. 10a). In contrast, $\mathrm{SiO}_{2}-\mathrm{FeOt} / \mathrm{MgO}$ plot portrayed that these xenoliths and Tanawal Formation are calc-alkaline in nature, and some of the samples reveal tholeiitic behavior (Fig. 10b). Based on $\mathrm{A} / \mathrm{CNK}$ vs. ANK, $\mathrm{SiO}_{2}$ vs. $\mathrm{K}_{2} \mathrm{O},\left(\mathrm{Al}_{2} \mathrm{O}_{3}+\mathrm{CaO}\right) / \mathrm{FeOt}+$ $\mathrm{Na}_{2} \mathrm{O}+\mathrm{K}_{2} \mathrm{O}$ vs. $100\left(\mathrm{MgO}+\mathrm{FeOt}+\mathrm{TiO}_{2}\right) / \mathrm{SiO}_{2}$ and $\mathrm{SiO}_{2}$ vs. $\mathrm{FeOt} / \mathrm{MgO}$, the geochemical data of $\mathrm{MG}$ reveals calc-alkaline and strongly peraluminous nature (Fig. 11a-d).

Further, chemical data of the xenoliths and Tanawal Formation plotted on geotectonic discrimination diagram displays that samples of xenoliths and Tanawal Formation fall in syn-collisional field of $\mathrm{R}_{1}$ - 
Table 2a. Major and minor oxides (percent) in xenoliths and Tanawal Formation

\begin{tabular}{|c|c|c|c|c|c|c|c|c|c|c|c|c|}
\hline Sample No. & Petrology & $\mathrm{SiO}_{2}$ & $\mathrm{Al}_{2} \mathrm{O}_{3}$ & $\mathrm{Fe}_{2} \mathrm{O}_{3}$ & $\mathrm{CaO}$ & $\mathrm{MgO}$ & $\mathrm{Na}_{2} \mathrm{O}$ & $\mathrm{K}_{2} \mathrm{O}$ & $\mathrm{P}_{2} \mathrm{O}_{5}$ & $\mathrm{TiO}_{2}$ & $\mathrm{MnO}$ & LOI \\
\hline MR-2 & Restite & 62.16 & 16.85 & 6.51 & 1.99 & 2.49 & 2.31 & 2.86 & 0.27 & 0.96 & 0.08 & 1.85 \\
\hline MR-3 & Restite & 62.53 & 15.9 & 5.52 & 2.67 & 2.07 & 2.61 & 2.28 & 0.31 & 0.86 & 0.07 & 1.83 \\
\hline MR-7 & Restite & 62.98 & 16.98 & 6.58 & 2.02 & 2.52 & 2.32 & 2.9 & 0.27 & 0.96 & 0.08 & 2.05 \\
\hline MR-12 & Restite & 60.76 & 16.81 & 5.53 & 2.93 & 2.37 & 2.74 & 2.43 & 0.3 & 0.74 & 0.07 & 1.88 \\
\hline MR-18 & Restite & 81.98 & 10.1 & 1.27 & 2.23 & 0.41 & 1.29 & 0.5 & 0.11 & 0.17 & 0.08 & 1.76 \\
\hline MR-20 & Restite & 60.8 & 18.32 & 5.26 & 0.81 & 1.01 & 1.24 & 6.1 & 0.07 & 0.76 & 0.08 & 2.09 \\
\hline MR-22 & Restite & 64.66 & 16.05 & 5.1 & 2.2 & 1.56 & 3.19 & 2.42 & 0.31 & 0.65 & 0.07 & 1 \\
\hline MR-23 & Restite & 64.4 & 17.54 & 4.98 & 0.77 & 1.68 & 1.28 & 4.63 & 0.08 & 0.66 & 0.07 & 2.03 \\
\hline MR-26 & Restite & 63.63 & 15.53 & 5.39 & 2.61 & 2.02 & 2.55 & 2.22 & 0.3 & 0.84 & 0.06 & 1.19 \\
\hline MR-29 & Restite & 61.76 & 18.19 & 5.23 & 0.8 & 1.03 & 1.26 & 6.11 & 0.07 & 0.76 & 0.08 & 2.63 \\
\hline MR-30 & Restite & 65.69 & 17.85 & 5.09 & 0.78 & 1.72 & 1.31 & 4.72 & 0.19 & 0.68 & 0.08 & 1.13 \\
\hline MR-31 & Restite & 62.63 & 17.32 & 4.73 & 0.6 & 1.54 & 1.03 & 7.38 & 0.12 & 0.71 & 0.07 & 1.33 \\
\hline MR-32 & Restite & 65.13 & 16.43 & 5.12 & 2.2 & 1.55 & 3.19 & 2.42 & 0.32 & 0.65 & 0.06 & 1.05 \\
\hline MT-34 & Tanawal Formation & 62.97 & 19.06 & 3.02 & 0.3 & 0.59 & 1.58 & 7.82 & 0.07 & 0.31 & 0.06 & 1.2 \\
\hline MT-35 & Tanawal Formation & 62.33 & 18.83 & 3.01 & 0.29 & 0.59 & 1.59 & 7.75 & 0.07 & 0.31 & 0.06 & 1.77 \\
\hline MR-38 & Restite & 59.91 & 16.34 & 6.13 & 1.5 & 2.57 & 3.04 & 3.09 & 0.25 & 0.53 & 0.1 & 2.51 \\
\hline MR-41 & Restite & 63.45 & 17.03 & 5.05 & 2.25 & 2.01 & 3.69 & 2.2 & 0.32 & 0.79 & 0.08 & 1.77 \\
\hline $\mathrm{MR}-42$ & Restite & 62.76 & 16.82 & 4.98 & 2.2 & 2.02 & 3.66 & 2.18 & 0.32 & 0.79 & 0.07 & 1.9 \\
\hline MR-43 & Restite & 61.73 & 17.08 & 5.62 & 2.98 & 2.4 & 2.78 & 2.47 & 0.31 & 0.75 & 0.07 & 2.37 \\
\hline MR-44 & Restite & 65.64 & 16.5 & 5.71 & 2.41 & 1.08 & 2.09 & 2.81 & 0.25 & 0.82 & 0.08 & 1.62 \\
\hline MR-45 & Restite & 65.33 & 17.73 & 5.02 & 0.75 & 1.69 & 1.31 & 4.68 & 0.19 & 0.68 & 0.08 & 1.66 \\
\hline MR-48 & Restite & 81.04 & 9.99 & 1.25 & 2.2 & 0.4 & 1.27 & 0.49 & 0.11 & 0.17 & 0.08 & 1.09 \\
\hline MR-50 & Restite & 65.86 & 16.35 & 5.19 & 2.24 & 1.59 & 3.25 & 2.47 & 0.32 & 0.66 & 0.07 & 1.56 \\
\hline MR-52 & Restite & 60.65 & 18.17 & 7.11 & 3.71 & 2.25 & 1.95 & 2.31 & 0.41 & 1.12 & 0.1 & 1.3 \\
\hline MR-54 & Restite & 63.88 & 16.11 & 5.02 & 2.16 & 1.52 & 3.13 & 2.38 & 0.31 & 0.63 & 0.06 & 2.36 \\
\hline MR-56 & Restite & 62.98 & 15.83 & 5.48 & 2.31 & 1.04 & 2.01 & 2.7 & 0.24 & 0.79 & 0.08 & 1.62 \\
\hline MR-57 & Restite & 62.5 & 16.14 & 3.38 & 2.18 & 1.69 & 3.97 & 2.02 & 0.24 & 0.48 & 0.07 & 2.3 \\
\hline MR-59 & Restite & 59.39 & 17.76 & 6.99 & 3.6 & 2.21 & 1.93 & 2.27 & 0.4 & 1.08 & 0.09 & 1.87 \\
\hline MR-60 & Restite & 62.02 & 15.73 & 5.44 & 2.63 & 2.03 & 2.56 & 2.23 & 0.3 & 0.84 & 0.05 & 1.67 \\
\hline MT-64 & Tanawal Formation & 64.88 & 16.47 & 5.7 & 2.75 & 2.12 & 2.69 & 2.34 & 0.32 & 0.88 & 0.06 & 1.6 \\
\hline MT-66 & Tanawal Formation & 78.59 & 9.67 & 1.24 & 2.08 & 0.38 & 1.22 & 0.47 & 0.11 & 0.17 & 0.08 & 1.51 \\
\hline MR-67 & Restite & 77.79 & 13.49 & 1.22 & 2.06 & 0.38 & 1.21 & 0.46 & 0.11 & 0.17 & 0.08 & 1.54 \\
\hline MR-68 & Restite & 60.63 & 16.94 & 5.71 & 2.26 & 1.48 & 3.29 & 2.3 & 0.33 & 0.62 & 0.08 & 2.64 \\
\hline MR-69 & Restite & 61 & 16.94 & 5.44 & 2.87 & 2.33 & 2.74 & 2.52 & 0.3 & 0.76 & 0.08 & 2.31 \\
\hline MR-72 & Restite & 62.71 & 17.48 & 5.61 & 2.96 & 2.41 & 2.82 & 2.6 & 0.31 & 0.78 & 0.08 & 1.9 \\
\hline MR-73 & Restite & 61.41 & 17.01 & 5.78 & 2.25 & 1.49 & 3.3 & 2.33 & 0.34 & 0.63 & 0.08 & 1.71 \\
\hline MR-74 & Restite & 64.81 & 16.65 & 3.49 & 2.25 & 1.74 & 4.1 & 2.08 & 0.25 & 0.5 & 0.07 & 1.52 \\
\hline MR-75 & Restite & 60.33 & 17.88 & 5.95 & 1.65 & 1.31 & 1.95 & 3.38 & 0.21 & 0.51 & 0.1 & 2.39 \\
\hline MR-78 & Restite & 61.81 & 18.35 & 6.1 & 1.69 & 1.33 & 1.98 & 3.45 & 0.21 & 0.55 & 0.11 & 1.99 \\
\hline
\end{tabular}


Table 2b. Trace elements (ppm) in xenoliths and Tanawal Formation

\begin{tabular}{|c|c|c|c|c|c|c|}
\hline Sample No. & Petrology & $\mathrm{Zr}$ & $\mathrm{La}$ & $\mathrm{Ce}$ & Th & $\mathrm{V}$ \\
\hline MR-2 & Restite & 149 & 33 & 16 & 27 & 6 \\
\hline MR-3 & Restite & 196 & 33 & 24 & 28 & 7 \\
\hline MR-7 & Restite & 71 & 25 & 8 & 26 & 7 \\
\hline MR-12 & Restite & 148 & 30 & 11 & 28 & 4 \\
\hline MR-18 & Restite & 74 & 32 & 9 & 13 & 9 \\
\hline MR-20 & Restite & 75 & 18 & 12 & 24 & 12 \\
\hline MR-22 & Restite & 122 & 25 & 16 & 17 & 14 \\
\hline MR-23 & Restite & 120 & 29 & 15 & 14 & 8 \\
\hline MR-26 & Restite & 74 & 19 & 8 & 26 & 6 \\
\hline MR-29 & Restite & 194 & 21 & 16 & 28 & 5 \\
\hline MR-30 & Restite & 76 & 28 & 9 & 21 & 9 \\
\hline MR-31 & Restite & 223 & 22 & 16 & 23 & 10 \\
\hline MR-32 & Restite & 122 & 26 & 12 & 25 & 8 \\
\hline MT-34 & Tanawal Formation & 146 & 29 & 15 & 22 & 22 \\
\hline MT-35 & Tanawal Formation & 77 & 27 & 18 & 19 & 4 \\
\hline MR-38 & Restite & 148 & 17 & 15 & 16 & 24 \\
\hline MR-41 & Restite & 76 & 21 & 9 & 13 & 5 \\
\hline MR-42 & Restite & 147 & 18 & 8 & 25 & 6 \\
\hline MR-43 & Restite & 148 & 24 & 11 & 27 & 7 \\
\hline MR-44 & Restite & 120 & 20 & 7 & 14 & 9 \\
\hline MR-45 & Restite & 147 & 19 & 13 & 27 & 3 \\
\hline MR-48 & Restite & 196 & 23 & 14 & 29 & 5 \\
\hline MR-50 & Restite & 148 & 25 & 12 & 18 & 6 \\
\hline MR-52 & Restite & 149 & 22 & 9 & 15 & 10 \\
\hline MR-54 & Restite & 122 & 28 & 12 & 21 & 8 \\
\hline MR-56 & Restite & 120 & 13 & 17 & 23 & 12 \\
\hline MR-57 & Restite & 148 & 17 & 18 & 20 & 8 \\
\hline MR-59 & Restite & 145 & 15 & 19 & 18 & 9 \\
\hline MR-60 & Restite & 196 & 19 & 22 & 16 & 11 \\
\hline MT-64 & Tanawal Formation & 148 & 22 & 12 & 25 & 5 \\
\hline MT-66 & Tanawal Formation & 74 & 21 & 9 & 18 & 8 \\
\hline MR-67 & Restite & 74 & 24 & 8 & 15 & 4 \\
\hline MR-68 & Restite & 72 & 18 & 10 & 18 & 6 \\
\hline MR-69 & Restite & 148 & 8 & 17 & 14 & 12 \\
\hline MR-72 & Restite & 74 & 16 & 12 & 19 & 9 \\
\hline MR-73 & Restite & 124 & 23 & 16 & 24 & 7 \\
\hline MR-74 & Restite & 148 & 21 & 13 & 21 & 6 \\
\hline MR-75 & Restite & 121 & 19 & 15 & 26 & 10 \\
\hline MR-78 & Restite & 149 & 24 & 21 & 19 & 9 \\
\hline
\end{tabular}


Table 3a. Major and minor oxides (percent) in Mansehra Granite

\begin{tabular}{|c|c|c|c|c|c|c|c|c|c|c|c|c|c|}
\hline Sample No. & Petrology & $\mathrm{SiO}_{2}$ & $\mathrm{~K}_{2} \mathrm{O}$ & $\mathrm{Na}_{2} \mathrm{O}$ & $\mathrm{CaO}$ & $\mathrm{Al}_{2} \mathrm{O}_{3}$ & $\mathrm{Fe}_{2} \mathrm{O}_{3}$ & $\mathrm{MgO}$ & $\mathrm{FeO}$ & $\mathrm{TiO}_{2}$ & $\mathrm{MnO}$ & $\mathrm{P}_{2} \mathrm{O}_{5}$ & LOI \\
\hline MG-06 & Massive Granite & 70.03 & 4.07 & 4.17 & 1.21 & 15.45 & 0.83 & 1.49 & 2.15 & 0.78 & 0.07 & 0.29 & 0.21 \\
\hline MG-07 & Massive Granite & 71.15 & 3.73 & 4.05 & 1.39 & 15.11 & 0.85 & 1.19 & 1.75 & 0.57 & 0.04 & 0.23 & 0.33 \\
\hline MG-08 & Massive Granite & 71.7 & 3.16 & 3.15 & 1.61 & 14.08 & 2.47 & 1.19 & 1.16 & 0.57 & 0.09 & 0.3 & 0.65 \\
\hline MG-09 & Massive Granite & 72.06 & 4.25 & 2.65 & 1.31 & 16.25 & 0.43 & 0.59 & 1.71 & 0.45 & 0.02 & 0.19 & 0.25 \\
\hline MG-10 & Massive Granite & 73.15 & 3.75 & 2.78 & 1.14 & 15.33 & 0.44 & 0.73 & 1.46 & 0.43 & 0.12 & 0.17 & 0.34 \\
\hline MG-11 & Massive Granite & 68.75 & 4.17 & 3.16 & 1.22 & 15.39 & 1.59 & 0.79 & 1.29 & 0.43 & 0.08 & 0.23 & 2.04 \\
\hline MG-12 & Massive Granite & 71.02 & 3.17 & 2.59 & 1.31 & 14.23 & 0.68 & 0.79 & 2.79 & 0.41 & 0.07 & 0.18 & 2.05 \\
\hline MG-13 & Massive Granite & 71.16 & 2.49 & 3.49 & 0.7 & 16.27 & 0.18 & 1.85 & 1.18 & 0.66 & 0.04 & 0.23 & 1.03 \\
\hline MG-14 & Massive Granite & 70.35 & 2.43 & 3.14 & 1.59 & 15.63 & 2.13 & 1.41 & 0.87 & 0.65 & 0.06 & 0.25 & 0.95 \\
\hline MG-15 & Massive Granite & 69.95 & 3.23 & 3.05 & 1.53 & 14.57 & 2.15 & 1.29 & 1.29 & 0.59 & 0.14 & 0.22 & 1.83 \\
\hline MG-16 & Massive Granite & 71.05 & 3.57 & 3.03 & 1.41 & 14.03 & 0.52 & 0.89 & 1.81 & 0.43 & 0.07 & 0.16 & 2.41 \\
\hline MG-17a & Massive Granite & 73.79 & 2.69 & 2.27 & 1.25 & 15.79 & 0.69 & 0.77 & 1.03 & 0.29 & 0.09 & 0.17 & 1.02 \\
\hline MG-17b & Massive Granite & 72.65 & 3.89 & 2.79 & 1.27 & 15.29 & 0.43 & 0.89 & 1.23 & 0.49 & 0.07 & 0.16 & 0.87 \\
\hline MG-19 & Massive Granite & 69.03 & 4.85 & 3.73 & 1.03 & 17.55 & 0.58 & 0.85 & 1.03 & 0.37 & 0.09 & 0.25 & 0.63 \\
\hline MG-23 & Sheared Granite & 70.28 & 2.43 & 4.15 & 0.63 & 17.93 & 0.13 & 0.26 & 1.67 & 0.65 & 0.07 & 0.28 & 1.53 \\
\hline MG-27 & Sheared Granite & 73.06 & 3.55 & 3.03 & 1.13 & 15.49 & 1.31 & 0.59 & 1.69 & 0.35 & 0.06 & 0.18 & 0.26 \\
\hline MG-28 & Sheared Granite & 73.39 & 3.49 & 3.01 & 1.15 & 15.65 & 1.3 & 0.64 & 0.91 & 0.34 & 0.08 & 0.21 & 0.34 \\
\hline MG-30a & Sheared Granite & 70.75 & 5.28 & 2.78 & 1.12 & 14.19 & 1.19 & 0.57 & 1.03 & 0.31 & 0.16 & 0.16 & 2.35 \\
\hline MG-30b & Sheared Granite & 68.35 & 3.5 & 2.63 & 1.57 & 15.13 & 2.5 & 1.29 & 1.9 & 0.69 & 0.06 & 0.17 & 1.87 \\
\hline MG-33 & Sheared Granite & 69.85 & 4.43 & 3.32 & 0.89 & 15.23 & 0.34 & 0.69 & 1.28 & 0.34 & 0.08 & 0.18 & 2.65 \\
\hline MG-40 & Sheared Granite & 68.75 & 4.59 & 2.65 & 1.61 & 16.59 & 1.85 & 1.13 & 2.03 & 0.58 & 0.09 & 0.23 & 0.27 \\
\hline MG-42 & Sheared Granite & 70.85 & 4.36 & 2.53 & 1.23 & 14.29 & 0.31 & 0.61 & 1.89 & 0.34 & 0.12 & 0.12 & 2.63 \\
\hline MG-43 & Sheared Granite & 67.5 & 3.75 & 3.73 & 1.5 & 16.89 & 0.79 & 0.93 & 1.75 & 0.43 & 0.11 & 0.26 & 1.83 \\
\hline MG-44 & Sheared Granite & 69.55 & 3.65 & 3.48 & 1.09 & 15.24 & 0.74 & 0.86 & 1.85 & 0.42 & 0.06 & 0.25 & 2.11 \\
\hline MG-46 & Sheared Granite & 68.5 & 3.69 & 2.5 & 1.73 & 15.32 & 0.76 & 1.16 & 1.89 & 0.51 & 0.05 & 0.23 & 2.69 \\
\hline MG-49 & Massive Granite & 73.09 & 3.79 & 3.59 & 0.43 & 15.27 & 0.13 & 0.23 & 1.59 & 0.59 & 0.07 & 0.26 & 0.88 \\
\hline MG-64 & Massive Granite & 73.17 & 3.35 & 0.25 & 1.07 & 16.08 & 0.89 & 1.6 & 0.86 & 0.89 & 0.06 & 0.05 & 1.13 \\
\hline MG-65 & Massive Granite & 72.25 & 3.13 & 3.13 & 1.47 & 14.29 & 1.23 & 2.03 & 1.03 & 0.65 & 0.13 & 0.39 & 0.41 \\
\hline MG-67 & Massive Granite & 68.75 & 4.03 & 3.19 & 1.23 & 14.13 & 1.41 & 1.89 & 1.88 & 0.79 & 0.09 & 0.35 & 1.89 \\
\hline MG-68 & Massive Granite & 70.55 & 3.29 & 3.81 & 0.54 & 15.78 & 0.38 & 1.12 & 1.18 & 0.54 & 0.08 & 0.25 & 1.9 \\
\hline MG-69 & Massive Granite & 71.15 & 3.08 & 2.79 & 1.88 & 16 & 0.49 & 1.3 & 0.91 & 0.78 & 0.06 & 0.19 & 1.37 \\
\hline MG-70 & Massive Granite & 69.07 & 3.12 & 2.89 & 1.79 & 17.8 & 0.66 & 1.39 & 1.25 & 0.77 & 0.05 & 0.23 & 1.14 \\
\hline MG-71 & Massive Granite & 73.17 & 3.03 & 2.61 & 1.52 & 15.9 & 0.49 & 1.08 & 0.89 & 0.51 & 0.08 & 0.07 & 0.64 \\
\hline MG-72 & Massive Granite & 70.59 & 4.41 & 4.17 & 0.52 & 15.57 & 0.45 & 0.12 & 1.05 & 0.44 & 0.01 & 0.3 & 1.95 \\
\hline MG-73 & Massive Granite & 68.47 & 4.84 & 3.73 & 0.93 & 17.39 & 0.31 & 0.63 & 1.59 & 0.26 & 0.05 & 0.4 & 0.86 \\
\hline MG-74 & Massive Granite & 71.77 & 3.27 & 3.35 & 0.89 & 16.4 & 0.29 & 0.65 & 0.88 & 0.23 & 0.04 & 0.27 & 1.78 \\
\hline MG-75 & Massive Granite & 71.04 & 3.96 & 2.61 & 1.47 & 16.75 & 0.47 & 1.05 & 0.84 & 0.54 & 0.09 & 0.17 & 1.01 \\
\hline MG-76 & Massive Granite & 69.49 & 5.35 & 2.23 & 1.19 & 15.05 & 0.43 & 0.81 & 1.15 & 0.51 & 0.05 & 0.14 & 2.61 \\
\hline MG-77 & Massive Granite & 68.35 & 4.3 & 3.52 & 1.73 & 15.68 & 0.57 & 1.2 & 1.24 & 0.59 & 0.06 & 0.28 & 1.71 \\
\hline MG-78 & Massive Granite & 72.55 & 3.57 & 2.69 & 1.19 & 15.39 & 0.73 & 0.79 & 2.05 & 0.43 & 0.12 & 0.17 & 0.69 \\
\hline MG-79 & Massive Granite & 71.15 & 3.86 & 3.19 & 1.43 & 17.19 & 1.2 & 0.62 & 1.51 & 0.34 & 0.08 & 0.2 & 0.14 \\
\hline MG-80 & Massive Granite & 69.05 & 3.37 & 2.39 & 1.59 & 15.27 & 2.15 & 1.28 & 2.08 & 0.68 & 0.09 & 0.22 & 1.34 \\
\hline
\end{tabular}


Table 3b. Trace elements (ppm) in Mansehra Granite

\begin{tabular}{|c|c|c|c|c|c|c|c|c|c|c|c|c|}
\hline Sample No. & Petrology & $\mathrm{Y}$ & $\mathrm{Sr}$ & $\mathrm{Rb}$ & $\mathrm{Nb}$ & $\mathrm{Zr}$ & $\mathrm{U}$ & $\mathrm{Ba}$ & Th & $\mathrm{Ce}$ & $\mathrm{Nd}$ & $\mathrm{La}$ \\
\hline MG-06 & Massive Granite & 28 & 59 & 249 & 13 & 141 & 12 & 245 & 47 & 123 & 45 & 33 \\
\hline MG-07 & Massive Granite & 29 & 49 & 287 & 17 & 123 & 25 & 248 & 53 & 65 & 23 & 36 \\
\hline MG-08 & Massive Granite & 34 & 78 & 228 & 15 & 179 & 13 & 275 & 51 & 149 & 39 & 34 \\
\hline MG-09 & Massive Granite & 14 & 27 & 151 & 12 & 234 & 15 & 1621 & 39 & 89 & 2 & 37 \\
\hline MG-10 & Massive Granite & 30 & 47 & 263 & 15 & 121 & 11 & 259 & 50 & 155 & 21 & 45 \\
\hline MG-11 & Massive Granite & 28 & 58 & 253 & 16 & 146 & 14 & 277 & 45 & 112 & 21 & 39 \\
\hline MG-12 & Massive Granite & 26 & 56 & 235 & 13 & 125 & 12 & 206 & 52 & 147 & 42 & 46 \\
\hline MG-13 & Massive Granite & 10 & 38 & 21 & 18 & 166 & 13 & 92 & 55 & 112 & 7 & 11 \\
\hline MG-14 & Massive Granite & 31 & 67 & 279 & 17 & 143 & 23 & 249 & 49 & 72 & 39 & 29 \\
\hline MG-15 & Massive Granite & 27 & 55 & 243 & 13 & 129 & 16 & 348 & 48 & 66 & 3 & 52 \\
\hline MG-16 & Massive Granite & 25 & 63 & 247 & 16 & 127 & 14 & 279 & 53 & 118 & 28 & 39 \\
\hline MG-17a & Massive Granite & 17 & 73 & 125 & 11 & 104 & 14 & 239 & 43 & 92 & 4 & 49 \\
\hline MG-17b & Massive Granite & 29 & 64 & 235 & 14 & 125 & 15 & 351 & 46 & 143 & 36 & 46 \\
\hline MG-19 & Massive Granite & 33 & 61 & 261 & 14 & 151 & 15 & 281 & 55 & 133 & 27 & 48 \\
\hline MG-23 & Sheared Granite & 24 & 48 & 289 & 14 & 87 & 10 & 221 & 45 & 137 & 19 & 34 \\
\hline MG-27 & Sheared Granite & 33 & 75 & 199 & 15 & 165 & 13 & 349 & 55 & 125 & 29 & 47 \\
\hline MG-28 & Sheared Granite & 24 & 49 & 248 & 16 & 115 & 14 & 2429 & 41 & 165 & 27 & 32 \\
\hline MG-30a & Sheared Granite & 25 & 51 & 255 & 14 & 106 & 17 & 339 & 44 & 69 & 5 & 51 \\
\hline MG-30b & Sheared Granite & 19 & 162 & 139 & 17 & 124 & 11 & 211 & 45 & 189 & 26 & 36 \\
\hline MG-33 & Sheared Granite & 22 & 45 & 235 & 15 & 91 & 15 & 278 & 42 & 117 & 25 & 25 \\
\hline MG-40 & Sheared Granite & 27 & 76 & 227 & 17 & 161 & 13 & 371 & 53 & 139 & 53 & 47 \\
\hline MG-42 & Sheared Granite & 25 & 64 & 191 & 10 & 107 & 12 & 319 & 47 & 137 & 39 & 43 \\
\hline MG-43 & Sheared Granite & 21 & 41 & 225 & 15 & 99 & 11 & 179 & 42 & 125 & 25 & 29 \\
\hline MG-44 & Sheared Granite & 23 & 49 & 276 & 16 & 112 & 15 & 349 & 53 & 111 & 53 & 38 \\
\hline MG-46 & Sheared Granite & 19 & 46 & 326 & 14 & 88 & 24 & 181 & 47 & 65 & 18 & 27 \\
\hline MG-49 & Massive Granite & 23 & 73 & 185 & 11 & 149 & 13 & 362 & 48 & 88 & 28 & 23 \\
\hline MG-64 & Massive Granite & 32 & 75 & 186 & 14 & 178 & 14 & 261 & 49 & 75 & 18 & 31 \\
\hline MG-65 & Massive Granite & 34 & 61 & 235 & 16 & 156 & 10 & 235 & 52 & 73 & 5 & 47 \\
\hline MG-67 & Massive Granite & 25 & 56 & 231 & 15 & 134 & 13 & 238 & 52 & 12 & 5 & 33 \\
\hline MG-68 & Massive Granite & 18 & 8 & 372 & 17 & 25 & 11 & 23 & 33 & 63 & 17 & 13 \\
\hline MG-69 & Massive Granite & 47 & 105 & 206 & 21 & 276 & 21 & 532 & 54 & 110 & 19 & 53 \\
\hline MG-70 & Massive Granite & 34 & 75 & 191 & 18 & 185 & 18 & 415 & 49 & 74 & 32 & 37 \\
\hline MG-71 & Massive Granite & 43 & 63 & 205 & 19 & 312 & 21 & 366 & 63 & 123 & 38 & 61 \\
\hline MG-72 & Massive Granite & 11 & 9 & 192 & 9 & 15 & 25 & 28 & 34 & 23 & 13 & 12 \\
\hline MG-73 & Massive Granite & 32 & 35 & 345 & 19 & 89 & 24 & 182 & 49 & 69 & 36 & 23 \\
\hline MG-74 & Massive Granite & 24 & 48 & 243 & 12 & 105 & 25 & 265 & 44 & 55 & 32 & 18 \\
\hline MG-75 & Massive Granite & 31 & 59 & 209 & 19 & 181 & 21 & 229 & 57 & 125 & 47 & 42 \\
\hline MG-76 & Massive Granite & 32 & 56 & 219 & 18 & 161 & 24 & 325 & 51 & 53 & 23 & 37 \\
\hline MG-77 & Massive Granite & 5 & 15 & 47 & 6 & 32 & 23 & 55 & 34 & 48 & 7 & 14 \\
\hline MG-78 & Massive Granite & 27 & 54 & 261 & 15 & 119 & 12 & 279 & 51 & 127 & 42 & 33 \\
\hline MG-79 & Massive Granite & 22 & 51 & 181 & 8 & 83 & 17 & 235 & 43 & 92 & 8 & 31 \\
\hline MG-80 & Massive Granite & 31 & 86 & 205 & 17 & 204 & 15 & 325 & 56 & 97 & 21 & 27 \\
\hline
\end{tabular}



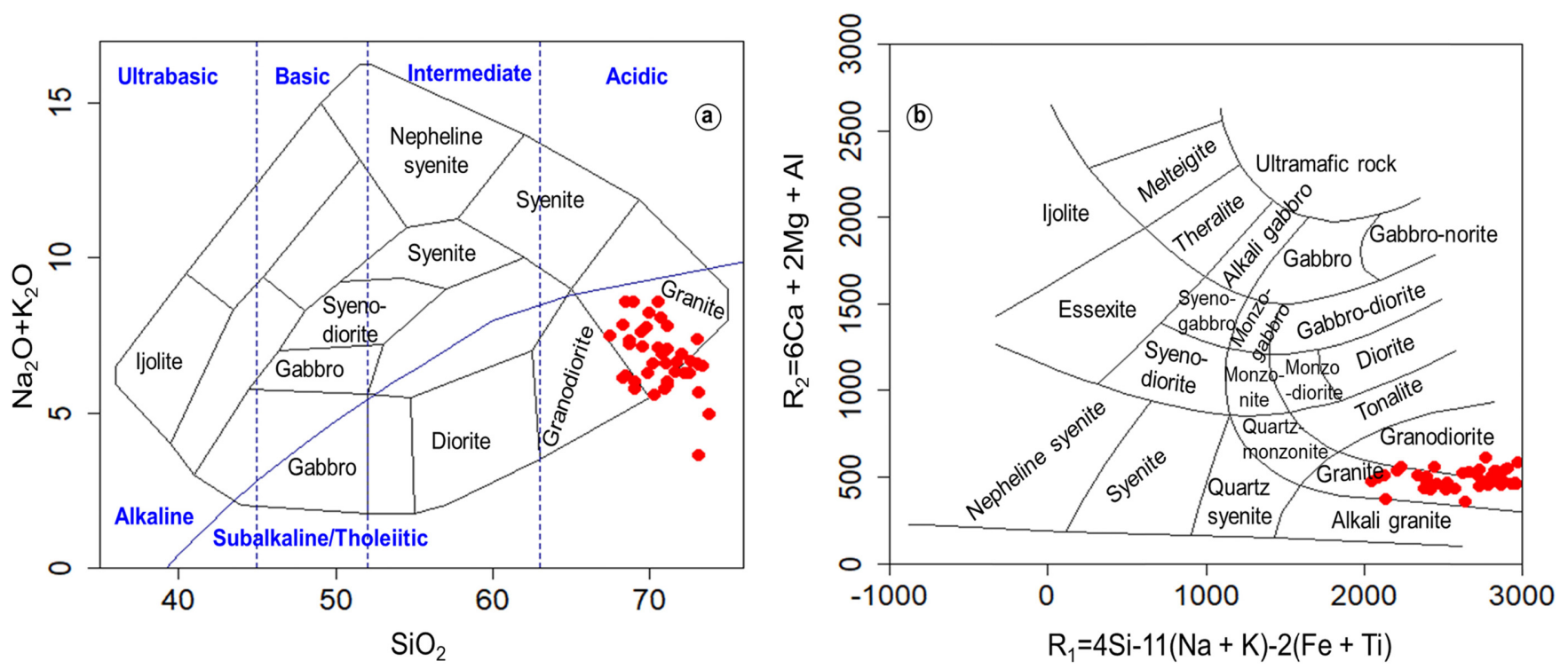

Figure 8. Binary plots of geochemical data of Mansehra Granite in geochemical classification diagrams indicating granitic fields (after Cox et al., 1979 and De la Roche et al., 1980); (a) $\mathrm{SiO}_{2} v$ s. total alkalis (b) Plot of $\mathrm{R}_{1} v$ s. $R_{2}$.
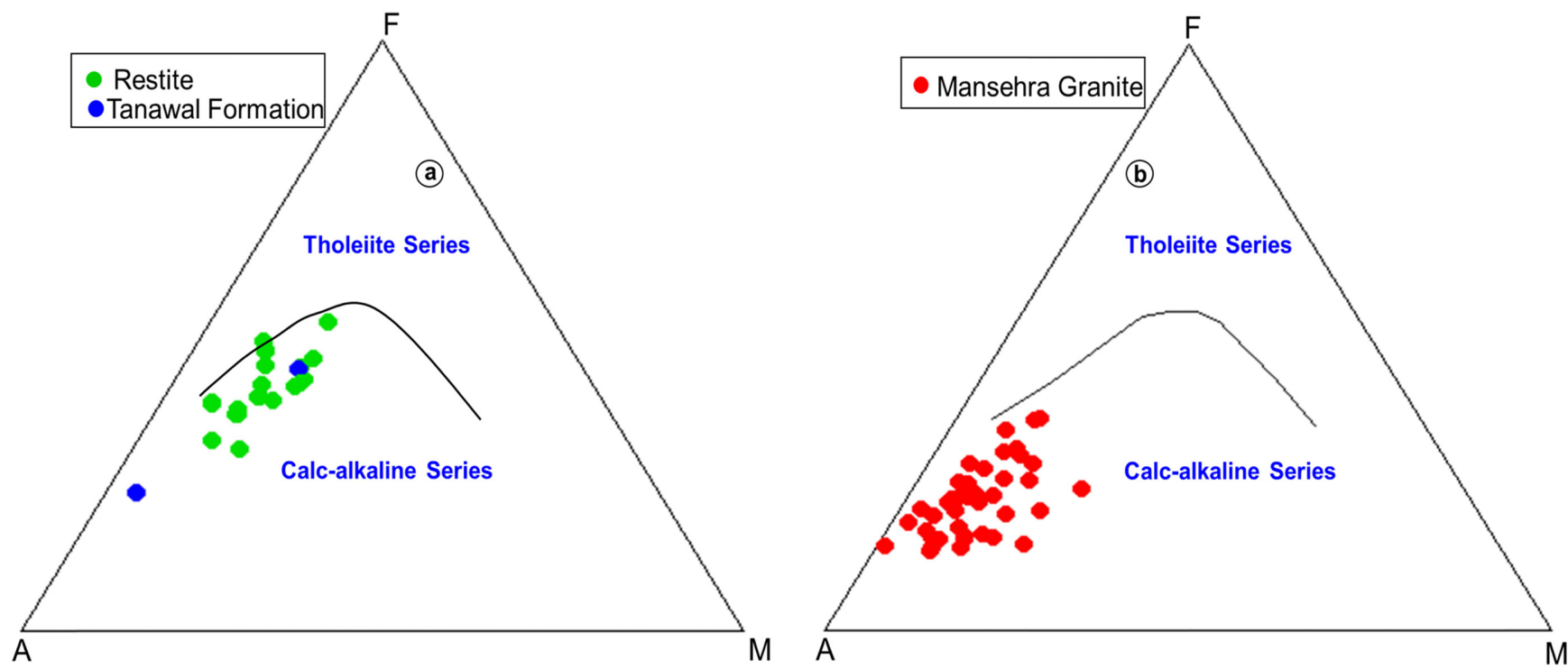

Figure 9. AFM diagram (Irvin and Baragar, 1971) of restites, Tanawal Formation and Mansehra Granite. (a) plot of restite and Tanawal Formation indicating their calc-alkaline character; (b) Mansehra Granite (MG) representing calc-alkaline nature.

$\mathrm{R}_{2}$ diagram (Fig. 12a). Likewise, geochemical data of the $\mathrm{MG}$ plotted on triangular tectonic discrimination diagrams denote syncollisional orogenic settings of the pluton (Fig. 12b-d).

Chemical data of xenoliths and Tanawal Formation plotted on Harker's variation diagrams express negative trends with $\mathrm{SiO}_{2}$ (Fig. 13ac). Geochemical plots of various oxides with $\mathrm{SiO}_{2}$ show scattering on account of irregular distribution of minerals and heterogeneity in the protolith (Fig. 13). Likewise, in variation diagrams of Mansehra Granite, major oxides also reveal negative correlation with $\mathrm{SiO}_{2}$ (Fig. 14). Meanwhile, the trace elements $\mathrm{Zr}, \mathrm{Sr}, \mathrm{Nb}, \mathrm{Y}$ and Th exhibit significant positive variations and relationships (Fig. 15a-d, f). Rb however, varied inversely with $\mathrm{Sr}$ (Fig. 15e) and exhibited negative correlation (Fig. 15e). These significant binary plots of major and trace elements describe scattering in Mansehra Granite.

In addition, petrographic observation and chemical data illustrate the presence of zircon in the xenoliths found in Mansehra Granite. Zircon saturation behaviour is a powerful tool to evaluate temperature of the xenoliths, metasediments and melts (Watson and Harrison, 1983; Montel, 1993; Miller et al., 2003; Janousek et al., 2006). Zircon saturation thermometry of both rocks determined through GCDKIT software shows that zircon temperatures of xenoliths and Tanawal Formation range from 734.8 to $850{ }^{\circ} \mathrm{C}$ and 753.1 to $808.6^{\circ} \mathrm{C}$, respectively. However, zircon saturation temperatures of the Mansehra Granite vary from 623.7 to $886.6{ }^{\circ} \mathrm{C}$. The temperature results are presented in Tables 4 and 5. 

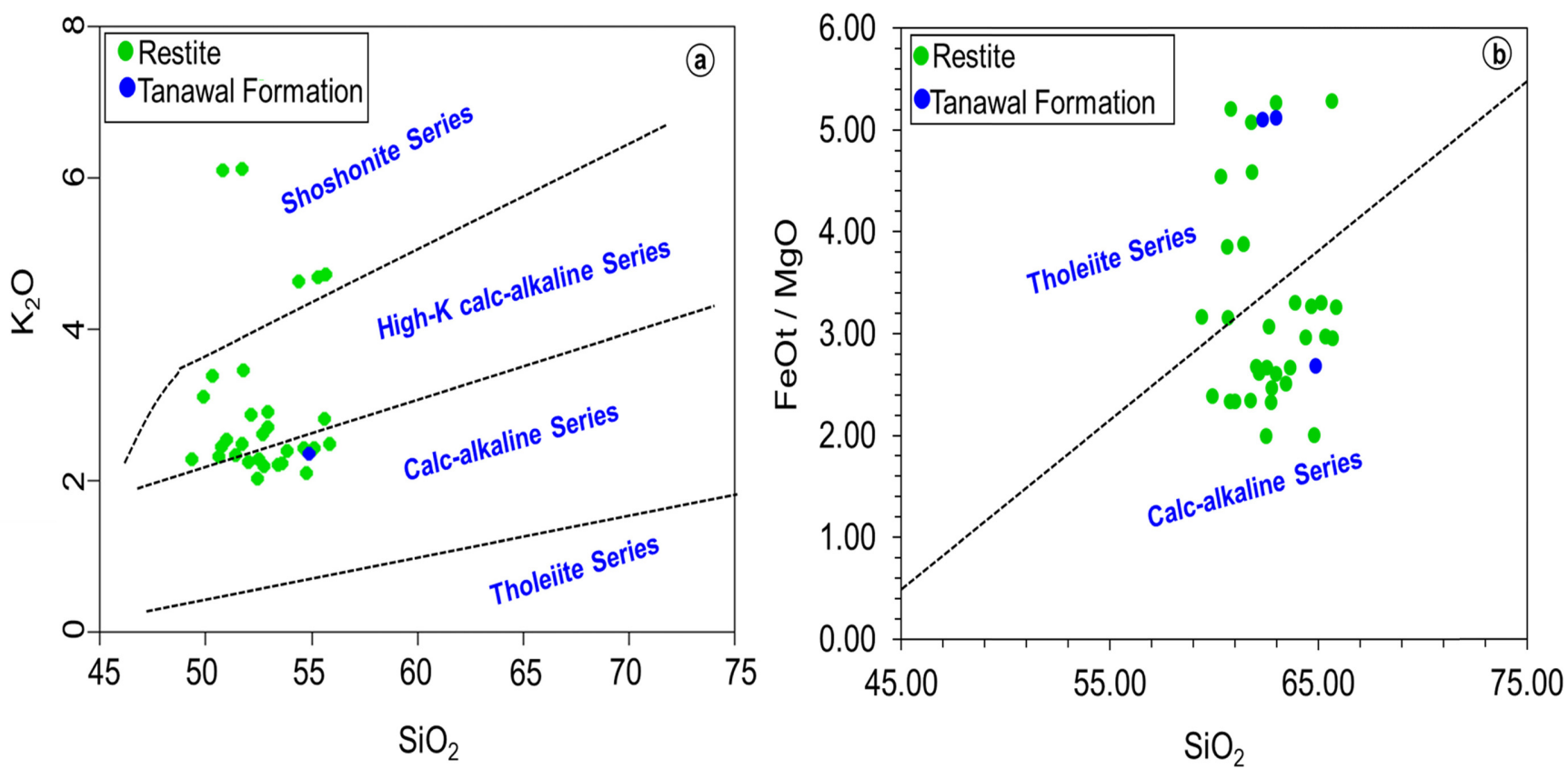

Figure 10. shows geochemical series of restites and Tanawal Formation: (a) $\mathrm{SiO}_{2}-\mathrm{K}_{2} \mathrm{O}$ plot (after Peccerillo and Taylor, 1976) indicating calcalkaline to High-K calc-alkaline nature; (b) $\mathrm{SiO}_{2}-\mathrm{FeOt/MgO}$ plot (after Miyashiro, 1974) exhibiting calc-alkaline to tholeiite series.
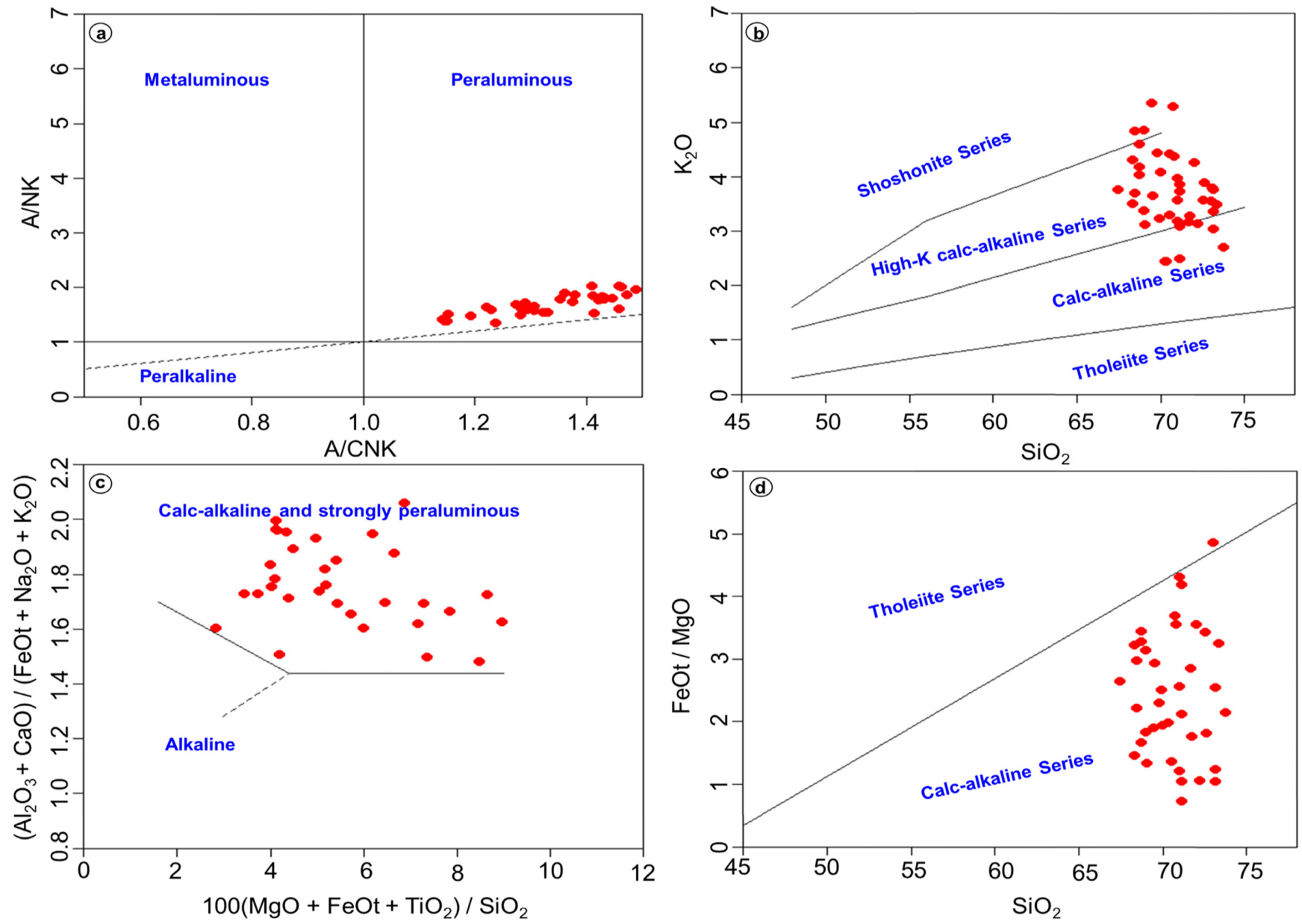

Figure 11. Plot of chemical data of Mansehra Granite reveals calc-alkaline and strongly peraluminous nature a) A/CNK vs. ANK (Shand, 1943); (b) $\mathrm{SiO}_{2}$ vs. $\mathrm{K}_{2} \mathrm{O}$ (after Peccerillo and Taylor, 1976); (c) $\left(\mathrm{Al}_{2} \mathrm{O}_{3}+\mathrm{CaO}\right) / \mathrm{FeOt}+\mathrm{Na}_{2} \mathrm{O}+\mathrm{K}_{2} \mathrm{O}$ vs. $100\left(\mathrm{MgO}+\mathrm{FeOt}^{+\mathrm{TiO}}\right.$ ) $/ \mathrm{SiO} \mathrm{O}_{2}(\mathrm{Sylvester}$, 1998); (d) $\mathrm{SiO}_{2}$ vs. FeOt/MgO (after Miyashiro, 1974). 

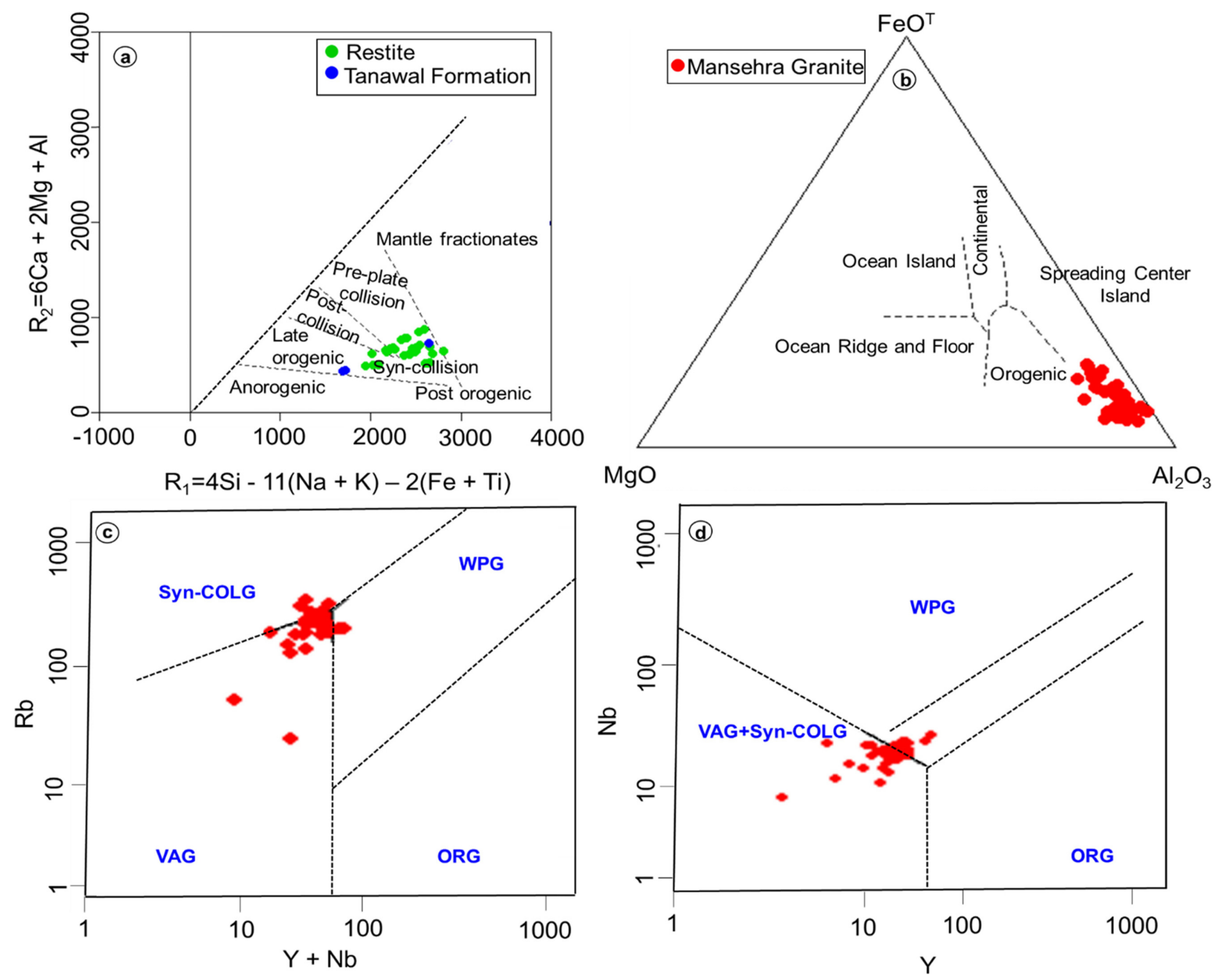

Figure 12. (a) $R_{1}-R_{2}$ diagram (Batchelor and Bowden, 1985) of xenoliths and Tanawal Formation showing orogenic syn-collisional settings; (b) Triangular Plot representing orogenic settings of Mansehra Granite (Pearce et al., 1977); (c, d) Tectonic discrimination diagrams signifying syn-collisional tectonic settings of Mansehra Granite (Pearce et al., 1984).

\section{Discussion}

\section{Field Characteristics and Geochemical Attributes of Xenoliths in Mansehra Granite}

Light-gray to jet-black xenoliths are found in Mansehra Granite in Oghi-Darband, Susalgali-Khaki, Chitta-Batta, Phulra and Mansehra areas. Light-gray xenoliths are non-foliated and contain relatively higher concentration of quartz as compared with mica contents and classified as micaceous quartzite. Whereas, light-gray xenoliths, which are principally comprised of quartz and feldspar with relatively lower mica contents, are classified as schistosed xenoliths (Fig. 3d, g). These schistosed xenoliths have tadpole-shaped K-feldspar phenocrysts with parallel alignment around their borders, suggesting the impact of stress and flow of Mansehra Granitic magma in a viscous state (Fig. 3d). Jet-black xenoliths contain relatively higher biotite contents as compared with dark-gray variety (Fig. 3b). These xenoliths are randomly distributed throughout the mass of Mansehra Granite which is contrary to the observation of Shams (1961) who proposed that these xenoliths are abundant near the contact of the granite with the Tanawal Formation.

Geochemically, lower $\mathrm{Na}_{2} \mathrm{O}$ values in xenoliths correspond to its lesser albite contents. The $\mathrm{K}_{2} \mathrm{O}$ constituents in xenoliths are $0.46-7.38 \%$, which relate to their modal $\mathrm{K}$-feldspar contents. On the other hand, lesser $\mathrm{K}_{2} \mathrm{O}$ level in xenoliths suggest lower modal microcline contents. The overall subordinate $\mathrm{CaO}$ and $\mathrm{Na}_{2} \mathrm{O}$ level relative to $\mathrm{K}_{2} \mathrm{O}$ represent lower modal plagioclase and higher microcline, whereas the smaller value of $\mathrm{MgO}$ is due to scarcity of chlorite. Relatively higher or lower levels of $\mathrm{TiO}_{2}$ and $\mathrm{MnO}$ in samples of xenoliths suggest augmentation or deficiency of biotite and garnet constituents.

\section{Implications on Petrogenesis and Tectonic Settings of Xenoliths}

Geochemical classification diagrams of light-gray to jet-black xenoliths and metasediments of the Tanawal Formation placed these rocks in calc-alkaline to high K calc-alkaline fields (Fig. 9a; 10a). Both xenoliths and metasediments of Tanawal Formation lie in syn-colli- 

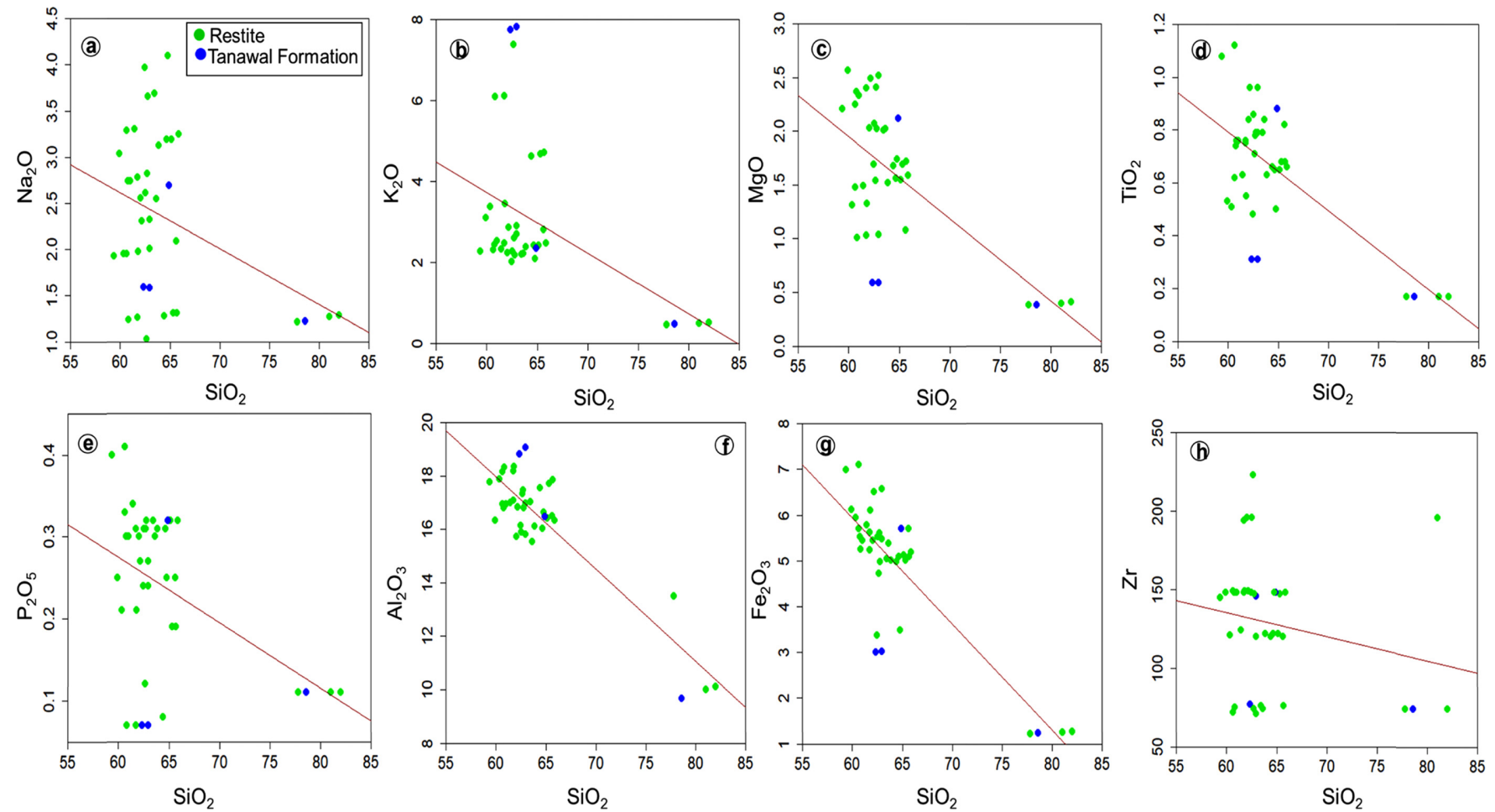

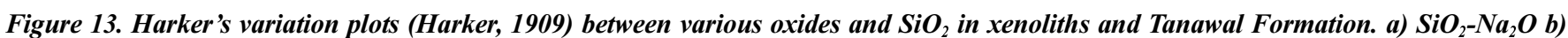
$\mathrm{SiO}_{2}-\mathrm{K}_{2} \mathrm{O}$ c) $\mathrm{SiO}_{2}-\mathrm{MgO}$ d) $\mathrm{SiO}_{2}-\mathrm{TiO}_{2}$ e) $\mathrm{SiO}_{2}-\mathrm{P}_{2} \mathrm{O}_{5}$ f) $\mathrm{SiO}_{2}-\mathrm{Al}_{2} \mathrm{O}_{3}$ g) $\left.\mathrm{SiO}_{2}-\mathrm{Fe}_{2} \mathrm{O}_{3} \mathrm{~h}\right) \mathrm{SiO}_{2}-\mathrm{Zr}$.
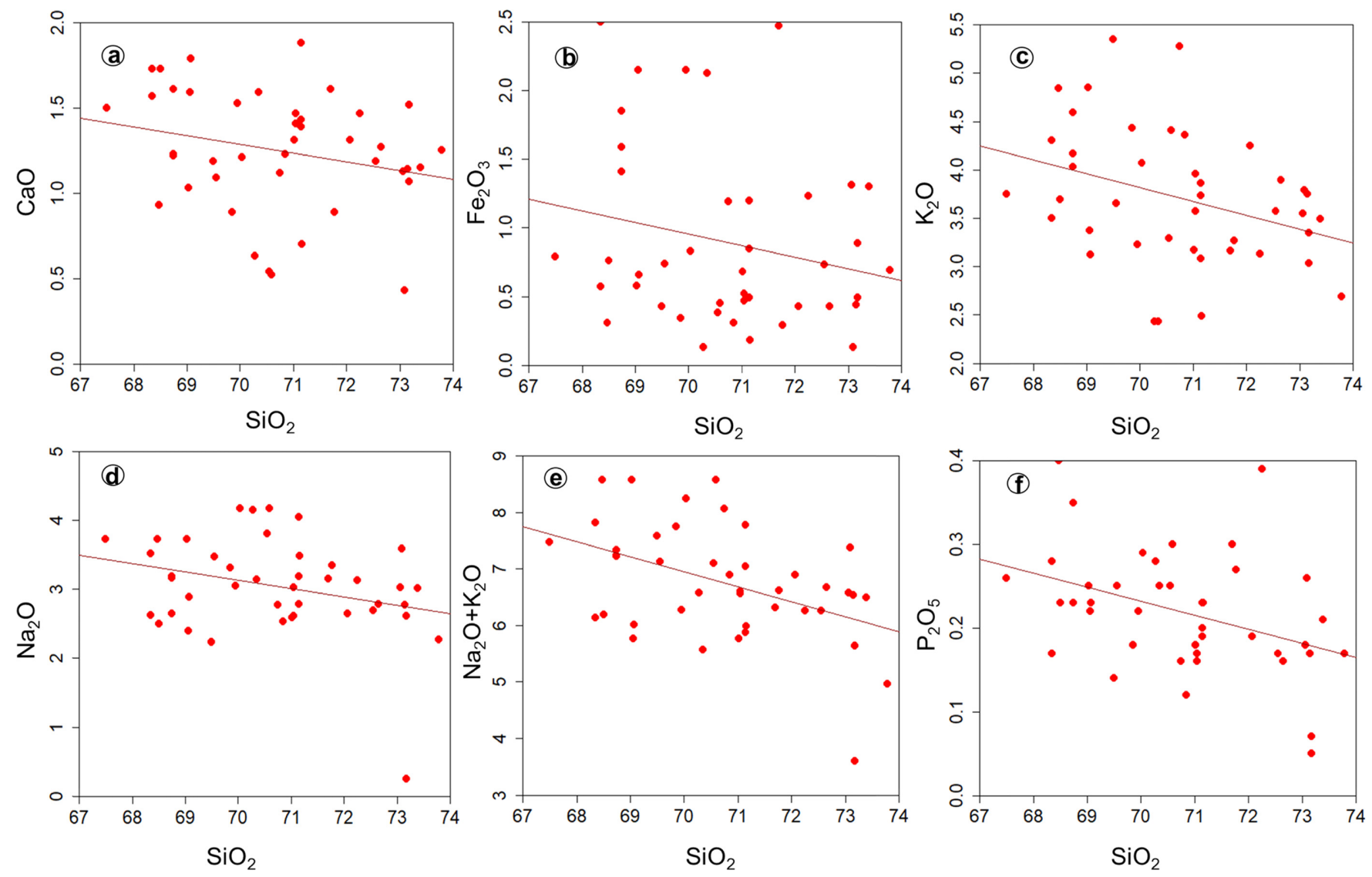

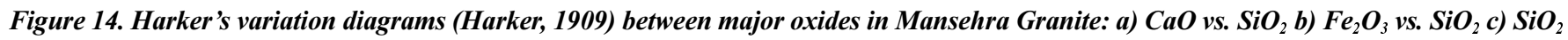
vs. $\mathrm{K}_{2} \mathrm{O}$ d) $\mathrm{Na}_{2} \mathrm{O}$ vs. $\mathrm{SiO}_{2}$ e) $\mathrm{SiO}_{2}$ vs. $\left.\mathrm{Na}_{2} \mathrm{O}+\mathrm{K}_{2} \mathrm{Of}\right) \mathrm{P}_{2} \mathrm{O}_{5}$ vs. $\mathrm{SiO}_{2}$. 

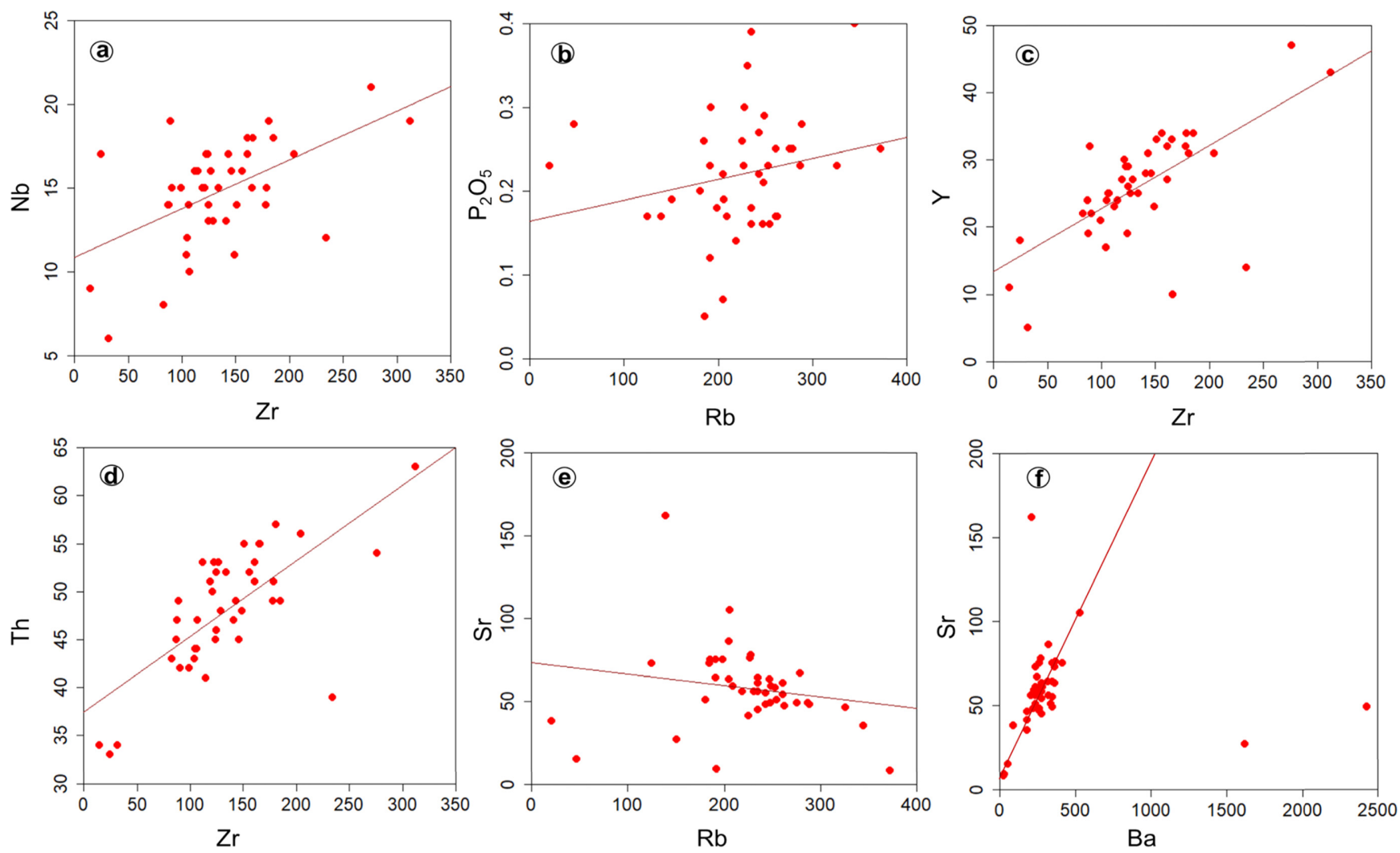

Figure 15. Harker's variation diagrams (Harker, 1909) between trace elements in Mansehra Granite: a) $\mathrm{Nb} v \mathrm{vs} . \mathrm{Zr}$ b) $\left.\mathrm{Rb} v \mathrm{~s} . \mathrm{P}_{2} \mathrm{O}_{5} \mathrm{c}\right) \mathrm{Zr}$ vs. $\mathrm{Y}$ d) Th vs. $\mathrm{Zr}$ e) $\mathrm{Rb} v \mathrm{~s} . \mathrm{Sr} f$ ) $\mathrm{Sr}$ vs. Ba.

sional orogenic domain of the tectonic discrimination diagrams (Fig. 12a), implying that these rocks are linked with orogenic syn-collision tectonic settings. In the meantime, orogenic syn-collisional tectonic setting in tectonic discrimination diagram also suggests that petrogenesis of these rocks is associated with compressional regime. Likewise, both these rocks have comparable modal mineralogical and geochemical composition (Tables 1 and 2). The light-gray to jet-black xenoliths and Tanawal Formation shared common features in variation diagrams (Fig. 13). These rocks also have comparable zircon saturation temperatures (Table 4). In view of the resemblance of geochemical signatures, geotectonic features and petrographic characteristics of the xenoliths and the metasediments of the Tanawal Formation, it can be inferred that the former has been derived from the later. These xenoliths now may be referred to as restites, which substantiate the hypothesis of anatectic nature and S-type trait of the Mansehra Granite. Occurrence of zircon and apatite in schistosed xenoliths is pointer of restites (Pupin, 1980; Pitcher, 1993). Enclosure of apatite and zircon in non-foliated micaceous quartzite xenoliths of the Mansehra Granite suggests the restite nature of these rocks. Meanwhile, presence of surmicaceous enclaves in Mansehra Granite also proposed the restite character of these rocks, which is similar to the findings of White et al. (1991).

Metasedimentary xenoliths present in S-type Mansehra Granite are restites which are analogous to conclusions of White et al. (1991) and Wyborn et al. (1991). Lack of sillimanite in metasedimentary xenoliths of S-type granite is pointer of restites (Clemes and Wall, 1981). The absence of sillimanite in schistosed rocks of the Mansehra area proposed the restite nature of xenoliths. In addition, xenoliths of Stype granites of the Lachlan fold belt, Australia deliver confirmation of the restite and these S-type granites are formed by anatexis of enclosing metasedimentary protolith (Chappell and White, 1974; Thompson, 1982; Le Fort et al., 1986; Clemens and Vielzeuf, 1987; Barbey et al., 1990; Holtz and Barbey, 1991; Inger and Harris, 1993; Albarede, 2003; Best, 2003). Likewise, we propose that the xenoliths of S-type Mansehra granites provide evidences of the restite and the Mansehra pluton is developed by anatexis of enclosing metasedimentary Tanawal Formation which is also consistent with the findings of Jan et al. (1981). In view of these findings, it can be inferred that the anatectic nature and S-type character of the Mansehra Granite suggests the enclosing Tanawal Formation as the probable protolith for the granitic melt of the pluton. The restite nature of the xenoliths found in the granite furnishes credible evidence in favour of the generation of Mansehra Granitic magma through partial melting of metasediments of the Tanawal Formation.

Association of larger biotite flakes at the contact of these restites with Mansehra Granite (Fig. 5d) points out that these restites represents non-melted fragments of the Tanawal Formation which were exposed to relatively higher temperature during anatexis of the protolith. Mean crystallization temperature of the Mansehra Granite ranged from 623.7 to $886.6{ }^{\circ} \mathrm{C}$. The restites most probably exposed to these higher temperatures, particularly at the margins and induced recrystallization and relatively larger flake size as compared with the central part of these restites due to lesser impact of elevated temperature.

Schistosed restites are abundant and exhibits conspicuous lepidoblastic texture having discernible symmetrical and asymmetrical crenulation cleavage (Fig. 5g). These restites are surmicaceous containing zircon and apatite (Fig. 5a-g). Schistosed restites record defor- 
Table 4. Zircon saturation temperatures of restites (MR) and Tanawal Formation (MT) samples from the Mansehra area

\begin{tabular}{|c|c|}
\hline Sample No. & Zr Saturation Temperature ${ }^{\circ} \mathrm{C}$ \\
\hline MR-2 & 807.9 \\
\hline MR-3 & 821.4 \\
\hline MR-7 & 744.5 \\
\hline MR-12 & 791.7 \\
\hline MR-18 & 762.0 \\
\hline MR-20 & 757.3 \\
\hline MR-22 & 779.3 \\
\hline MR-23 & 808.3 \\
\hline MR-26 & 739.1 \\
\hline MR-29 & 841.7 \\
\hline MR-30 & 768.3 \\
\hline MR-31 & 846.8 \\
\hline MR-32 & 781.2 \\
\hline MT-34 & 808.6 \\
\hline MT-35 & 753.1 \\
\hline MR-38 & 799.2 \\
\hline MR-41 & 737.9 \\
\hline MR-42 & 793.0 \\
\hline MR-43 & 791.7 \\
\hline MR-44 & 788.5 \\
\hline MR-45 & 827.2 \\
\hline MR-48 & 850.0 \\
\hline MR-50 & 796.1 \\
\hline MR-52 & 797.3 \\
\hline MR-54 & 781.0 \\
\hline MR-56 & 788.4 \\
\hline MR-57 & 789.7 \\
\hline MR-59 & 794.9 \\
\hline MR-60 & 822.3 \\
\hline MT-64 & 796.6 \\
\hline MT-66 & 763.3 \\
\hline MR-67 & 778.1 \\
\hline MR-68 & 736.1 \\
\hline MR-69 & 792.5 \\
\hline MR-72 & 734.8 \\
\hline MR-73 & 781.3 \\
\hline MR-74 & 789.8 \\
\hline MR-75 & 798.6 \\
\hline MR-78 & 817.9 \\
\hline
\end{tabular}

Table 5. Zircon saturation temperatures of Mansehra Granite (MG) from Mansehra area

\begin{tabular}{|c|c|}
\hline Sample No. & Zr Saturation Temperature ${ }^{\circ} \mathrm{C}$ \\
\hline MG-06 & 779.1 \\
\hline MG-07 & 768.8 \\
\hline MG-08 & 810.5 \\
\hline MG-09 & 850.2 \\
\hline MG-10 & 790.3 \\
\hline MG-11 & 794.9 \\
\hline MG-12 & 792.1 \\
\hline MG-13 & 828.6 \\
\hline MG-14 & 804.5 \\
\hline MG-15 & 785.2 \\
\hline MG-16 & 781.8 \\
\hline MG-17a & 792.7 \\
\hline MG-17b & 789.7 \\
\hline MG-19 & 797.5 \\
\hline MG-23 & 770.6 \\
\hline MG-27 & 817 \\
\hline MG-28 & 786.3 \\
\hline MG-30a & 759.8 \\
\hline MG-30b & 785.9 \\
\hline MG-33 & 755.3 \\
\hline MG-40 & 805.5 \\
\hline MG-42 & 771.1 \\
\hline MG-43 & 760.1 \\
\hline MG-44 & 773.8 \\
\hline MG-46 & 756.9 \\
\hline MG-49 & 807.8 \\
\hline MG-64 & 865.5 \\
\hline MG-65 & 801.8 \\
\hline MG-67 & 779.7 \\
\hline MG-68 & 667.4 \\
\hline MG-69 & 864.6 \\
\hline MG-70 & 831.2 \\
\hline MG-71 & 886.6 \\
\hline MG-72 & 623.7 \\
\hline MG-73 & 753 \\
\hline MG-74 & 782.2 \\
\hline MG-75 & 827.6 \\
\hline MG-76 & 805.6 \\
\hline MG-77 & 666.2 \\
\hline MG-78 & 790.3 \\
\hline MG-79 & 754.9 \\
\hline MG-80 & 836.5 \\
\hline
\end{tabular}


mations in the form of crenulations (Fig. 5g), and these metasedimentary restites preserve confirmation of structural antiquity at the time of their amalgamation in the felsic melt which is parallel to the conclusions of Fleming (1996).

In summary, this study suggests that these light-gray to jet-black restites have been derived from the Tanawal Formation, which is the protolith of the Mansehra Granite. Furthermore, these restites comprise fairly high $\mathrm{SiO}_{2}$ contents comparable to Tanawal Formation, reflecting that the restites are derived from metasediments of Tanawal Formation. Field relationships coupled with modal mineralogical composition, geochemical signatures and geotectonic association of the restites and the metasediments of the Tanawal Formation provide credible evidence in favour of anatectic nature and S-type trait of the Mansehra Granite.

\section{Petrogenetic and Tectonic Implications of Mansehra Granite}

Based on petrographic studies, the host rock of the restites (Mansehra Granite) represents abundance of Al-rich minerals specifically muscovite (Fig. 5j) and absence of allanite and titanite suggesting anatectic and S-type character of this pluton which is consistent with the interpretations of Villaseca et al. (2007) and Saki (2010). Apart from this, the geochemical plotting constructed for the Mansehra Granite (MG) placed this pluton in strongly peraluminous and calc-alkaline domain (Fig. 11a-d), which also imply anatectic and S-type trait of the Mansehra pluton. Meanwhile, the sheared and massive granites are plotted in the identical area of geochemical diagrams, representing that the both varieties of pluton are generated from similar compositional phase, therefore, can be reflected as cogenetic (Fig. 8, 9b, 11a-d). Triangular plot (Pearce et al., 1977) and trace elements plots particularly of $\mathrm{Y}-\mathrm{Nb}$ and $\mathrm{Y}+\mathrm{Nb}-\mathrm{Rb}$ (Pearce et al., 1984) denote that the samples of Mansehra pluton are clustered around triple point confluence of syn-COLG, VAG and WPG presenting orogenic syncollisional tectonic settings (Fig. 12b-d). Therefore, it can be deduced from these diagrams that the Mansehra pluton is linked with orogenic and syncollisional settings. In terms of trace element geochemistry, the deficiency of $\mathrm{Ba}$ and $\mathrm{Sr}$ in MG may signify robust partitioning of Ba into feldspar and micas and $\mathrm{Sr}$ into feldspar which is consistent with the interpretation of Icenhower and London (1996) and Blundry and Wood (1991). Meanwhile, extremely greater concentration of $\mathrm{Ba}$ in number of samples possibly can be ascribed to local enrichment. Fairly greater concentrations of $U(10-25 \mathrm{ppm})$ and $\mathrm{Th}(33-63 \mathrm{ppm})$ may propose that the MG is resulting from crystal fractionation of metasediments bearing Tanawal Formation, as argued by Azman (2005).

Harker's variation diagrams of the major oxides indicated negative correlations for Mansehra pluton (Fig. 14) which reflect crystallization of micas and feldspar in felsic melt of the granite. Parallel interpretations have also been demonstrated by Singh (2010). On the contrary, positive trend in the correlation of Ba vs. $\mathrm{Sr}$ (Fig. 15f) can probably depict feldspar fractionation in line with the conclusions testified by Janousek et al. (2002). Likewise, the positive relationship in plots of $\mathrm{Zr}$ with $\mathrm{Nb}$, Th, Y advocate Th, $\mathrm{Nb}$ and $\mathrm{Y}$ accumulation in crystallized zircon of MG (Fig. 15a, c-d). Geochemically, zircon crystallization in MG is preferred in peraluminous felsic melt comprising lower amounts of $\mathrm{CaO}$, which is parallel with the conclusions of Yurimoto et al. (1990).
Meanwhile, the decline of $\mathrm{Zr}$ and $\mathrm{P}_{2} \mathrm{O}_{5}$ with fractionation in MG most possibly exhibit zircon and apatite crystallization from the melt, as proposed by Nagudi et al. (2003). According to Chappell and White (1992), the positive correlation of $\mathrm{P}_{2} \mathrm{O}_{5}$ with $\mathrm{Rb}$ suggests that the felsic melt of granitic rock is resulting from metasediments. In the present study, the trend of $\mathrm{P}_{2} \mathrm{O}_{5}$ with $\mathrm{Rb}$ also exhibits positive correlation (Fig. 15b), implying that the felsic melt of Mansehra Granite is resulting from metasediments of Tanawal Formation. Therefore, in view of these grounds, it can be inferred that the silicic melt of the Mansehra pluton is resulting from Tanawal Formation. In contrast, the negative correlation of $\mathrm{Rb}$ with $\mathrm{Sr}$ (Fig. 15e) propose that during the MG melt evolution, fractional crystallization was possibly the key differentiation process in a close scheme, lacking significant contamination. This finding is comparable with the conclusions of Rapela and Shaw (1979). Based on these sights, it is worth stating that the plots of MG in variation diagrams indicate variability, reasonable correlations, and scattering in composition owing to heterogeneity in protolith (Tanawal Formation). Furthermore, the scattering in plots of major oxides may pertain to irregular minerals dissemination in the silicic melt of MG, as stated and testified by Sing and Kumar (2005).

\section{Conclusions}

Mansehra Granite contains light-gray to jet-black restites. Presence of zircon, apatite, surmicaceous nature and lack of sillimanite suggests that these rocks are restites which are erratically dispersed throughout the mass of Mansehra Granite rather than concentrated near the contact of the granite with the Tanawal Formation. Occurrence of abundant and relatively larger mica flakes at the contact of restites with Mansehra Granite revealed that due to exposure at higher temperature of the granitic melt prompted recrystallization and larger flake size as compared with the central part of restites owing to lesser impact of elevated temperature. Schistosed and surmicaceous restites depict crenulation cleavage which record deformations in the form of crenulations and preserve structural antiquity at the time of their amalgamation in the felsic melt.

Geochemical classification diagrams of light-gray to jet-black restites and metasediments of Tanawal Formation placed these rocks in calc-alkaline, tholeiitic and high K-calc-alkaline to shoshonite field and portrayed their compositional resemblance. The restites found in Mansehra Granite showed orogenic syn-collisional tectonic settings signifying their association with compressional regime. Similar mineralogical, geochemical composition and geotectonic signatures of lightgray to jet-black restites and metasediments of the Tanawal Formation represent derivation of the former from the later which reveals the anatectic nature and S-type character of the Mansehra Granite. Geochemical classification diagrams pertaining to massive and sheared Mansehra pluton place this granitic body in calc-alkaline domain with peraluminous S-type trait. Both, the sheared and massive S-type granites are generated from similar compositional phase, therefore, can be concluded as cogenetic. Trace elements plots of Mansehra pluton suggests association of MG with orogenic and syn-collisional settings. Careful interpretation of MG in Harker plots propose that the magma of this pluton was resulting from heterogeneous Tanawal Formation via process of fractional crystallization. 


\section{Acknowledgement}

This project was provided to Tehseen Zafar and greatly supported by grants of Institute of Geology, University of the Punjab Lahore, Pakistan. Authors are grateful to Editor-in-Chief Prof. Jin-Yong Lee and two anonymous reviewers for their constructive comments and suggestions.

\section{References}

Ashraf, M., and Chaudhry, M.N., 1976a, Geology and classification of Acid Minor bodies of Mansehra and Batgram area, Hazara division, Pakistan. Geological Bulletin of the Punjab University, v. 12, pp. 1-16.

Ashraf, M., and Chaudhry, M.N., 1976b, The geochemistry and petrogenesis of albitites from Mansehra and Batgram area, Hazara district, Pakistan. Geological Bulletin of the Punjab University, v. 13, pp. 65-85.

Ashraf, M., 1992, Petrogenesis of acid minor bodies of Mansehra Granitic Complex, Hazara Himalaya, NW Pakistan. Kashmir Journal of Geology, v. 10, pp. 1-26.

Albarede, F., 2003, Geochemistry: An Introduction. Cambridge University Press, United Kingdom (Cambridge University Press), 248 p.

Azman, A.G., 2005, Highly evolved S-type granite: Selim Granite, Main Rage Batholith, Peninsular Malaysia. Geological Society of Malaysia Bulletin, v. 51, pp. 95-101.

Barbey, P., MaCcaudiere, J., and Zzenti, J.P., 1990, High-pressure dehydration melting of metapelites: evidence from the migmatites of Yaoude (Cameroon). Journal of Petrology, v. 31, pp. 401-427.

Best, M.G., 2003, Igneous and Metamorphic petrology ( $2^{\text {nd }}$ edition). Blackwell Publishing, Oxford, United Kingdom, 752 p.

Batchelor, R.A., and Bowden, P., 1985, Petrogenetic interpretation of granitoid rock series using multicationic parameters. Chemical Geology, v. 48, pp. 43-55.

Bacon, C.R., 1986, Magmatic inclusions in silicic and intermediate rocks. Journal of Geophysical Research, v. 81, pp. 6091-6112.

Blundry, J.D., and Wood, B.J., 1991, Crystal chemical controls on the partitioning of $\mathrm{Sr}$ and $\mathrm{Ba}$ between plagioclase feldspar, silicate melts, and hydrothermal solutions. Geochimica et Cosmochimica Acta, v. 55, pp. 193-209.

Chappell, B.W., and White, A.J.R., 1974, Two contrasting granite types. Pacific Geology, v. 8, pp. 173-174.

Chappell, B.W., and White, A.J.R., 1992, I-Type and S-Type granites in the Lachlan Fold Belt. Transactions of the Royal Society of Edinburgh: Earth Sciences, v. 83, pp. 1-26.

Clemens, J.D., and Vielzeuf, D., 1987, Constraints on melting and magma production in the crust. Earth and Planetary Science Letters, v. 86, pp. 287-306.

Clemens, J.D., and Wall, V.J., 1981, Origin and crystallization of some peraluminous (S-type) granitic magmas. Contributions to Mineralogy and Petrology, v. 19, pp. 111-131.

Calkins, J.A., and Matin, A.A.S., 1968, The geology and the mineral resources of the Garhi Habibullah quadrangle and the Kakul area, Hazara district, West Pakistan. United States Department of Interiors, Geological Survey Project Report, Pakistan Investigation (IR), Pk-38.

Calkins, J.A., Offield, T.W., Abdullah, S.K., and Ali, S.T., 1975, Geology of the southern Himalaya in Hazara, Pakistan and Adjoining Areas. Geological Survey Professional Paper, vol. 716-C, pp. C1-C29.

Chaudhry, M.N., Ghazanfar, M., Ashraf, M., and Baloch, I.H., 1989, Observations on Precambrian orogeny and the age of the metamorphism in northwest Himalaya, Pakistan. Kashmir Journal of Geology, v. 6-7, pp. 19-22.

Cox, K.G., Bell, J.D., and Pankhurst, R.J., 1979, The interpretation of igneous rocks. George Allen and Unwin, London, $450 \mathrm{p}$.
De la Roche, H., Leterrier, J., Grandclaude, P., and Marchal, M., 1980, A classification of volcanic and plutonic rocks using R1-R2 diagrames and major-element analysis, its relationships with current nomenclature. Chemical Geology, v. 29, pp. 183-210.

Didier, J., and Barbarin, B., 1991, Enlcaves and Granite Petrology, Developments in Petrology. Elsevier, Amsterdam, v. 13, pp. 403-13.

Fleming, P.D., 1996, Inherited deformation structures in metasedimentary enclaves in granites as "windows" into deeper levels of the crust. Tectonophysics, v. 267, pp. 177-185.

Ganser, A., 1964, Geology of the Himalayas. Wiley Interscience, London, $289 \mathrm{p}$.

Holtz, F., and Barbey, P., 1991, Genesis of peraluminous granites II. Mineralogy and chemistry of the Tourem Complex (North Portugal). Sequential melting vs. restite unmixing. Journal of Petrology, v. 32, pp. 959-978.

Harker, A., 1909, The natural history of igneous rocks. Methuen, London, $384 \mathrm{p}$.

Icenhower, J.L., and London, D., 1996, Experimental partitioning of Rb, $\mathrm{Cs}, \mathrm{Sr}$ and Ba between alkali feldspar and peraluminous melt. American Mineralogist, v. 81, pp. 719-734.

Inger, S., and Harris, N., 1993, Geochemical constraints on leucogranites magmatism in the Langtang Valley, Nepal Himalaya. Journal of Petrology, v. 34, pp. 345-368.

Hodges, K.V., 2000, Tectonics of the Himalaya and southern Tibet from two perspectives. Geological Society of America Bulleton, v. 112, pp. $324-350$

Honegger, K., Dietrich, V., Frank, W., Gansser, A., Thoni, M., and Trommsdorff, V., 1982, Magmatism and metamorphism in the Ladakh Himalayas (the Indus-Tsangpo suture zone). Earth and Planetary Science Letters, v. 60, pp. 253-292.

Irvin, T.M., and Baragar, W.R., 1971, A guide to the chemical classification of common volcanic rocks. Canadian Journal of Earth Sciences, v. 8, pp. 523-548.

Jan, M.Q., Asif, M., Tahirkheli, T., and Kamal, M., 1981, Tectonic subdivision of granitic rocks of north Pakistan. Geological Bulletin (University of Peshawar), v. 14, pp. 159-182.

Janousek, V., Vrana, S., and Erban, V., 2002, Petrology, geochemical character and petrogenesis of a Variscan post-orogenic granite: case study from the Sevetin Massif, Moldanubian Batholith, Southern Bohemia. Journal of the Czech Geological Society, v. 47/1-2, pp. 1-22.

Janousek, V., Farrow, C.M., and Erban, V., 2006, Interpretation of wholerock geochemical data in igneous geochemistry: introducing Geochemical Data Toolkit (GCDKit). Journal of Petrology, v. 47, pp. 12551259.

Le Fort, P., 1975, Himalayas: the collided Range-Present knowledge of the continental arc. American Journal of Science, v. 275-A, pp. 1-44.

Le Fort, P., Debon, F., Pecher, A., Sonet, J., and Vidal, P., 1986, The 500 Ma magmatic event in Alpine southern Asia, a thermal episode at Gondwana scale. Sciences de la Terre, Memories, v. 47, pp. 191-209.

Miller, C.F., McDowell, S.M., and Mapes, R.., 2003, Hot and cold granites? Implication of zircon saturation temperatures and preservations of inheritance. Geology, v. 31, pp. 529-532.

Miyashiro, A., 1974, Volcanic Rock Series in Island Arcs and Active Continental Margins. American Journal of Science, v. 274, pp. 321-355.

Montel, J.M., 1993, A model for monazite/melt equilibrium and application to the generation of granite magmas. Chemical Geology, v. 110, pp. 127-146.

Naeem, M., Burg, J.P., Ahmad, N., Chaudhry, M.N., and Khalid, P., 2016, $\mathrm{U}-\mathrm{Pb}$ zircon systematics of the Mansehra Granitic Complex: implications on the early Paleozoic orogenesis in NW Himalaya of Pakistan. Geosciences Journal, v. 20, pp. 427-447.

Nagudi, B., Koeberl, C., and Kurat, G., 2003, Petrography and geochemistry of the Signo granite, Ugenda, and implications for its origin. Journal of African Earth Sciences, v. 36, pp. 73-87.

Pearce, T.H., Gorman, B.E., and Birkett, T.C., 1977, The relationship between 
major element chemistry and tectonic environment of basic and intermediate volcanic rocks. Earth and Planetary Science Letters, v. 36, pp. $121-132$.

Pearce, J.A., Harris, N.B.W., and Tindle, A.G., 1984, Trace element discrimination diagrams for the tectonic interpretation of granitic rocks. Journal of Petrology, v. 25, pp. 956-983.

Peccerillo, A., and Taylor, S.R., 1976, Geochemistry of Eocene Calc-Alkaline Volcanic Rocks from the Kastamonu Area, Northern Turkey. Contributions to Mineralogy and Petrology, v. 58, pp. 63-81.

Paudel, L.P., and Arita, K., 2000, Tectonic and polymetamorphic history of the Lesser Himalaya in Central Nepal. Journal of Asian Earth Science, v. 18 , pp. 561-584.

Pitcher, W.S., 1993, The nature and origin of granitic rocks. Chapman and Hall, Glasgow, $321 \mathrm{p}$.

Pupin, J.P., 1980, Zircon and granite petrology. Contributions to Mineralogy and Petrology, v. 73, pp. 207-220.

Rapela, C.W., and Shaw, D.M., 1979, Trace and major element models of granitoids gneisses in the Pampean Ranges, Argentina. Geochimica et Cosmochimica Acta, v. 43, pp. 1117-1129.

Saki, A., 2010, Mineralogy, geochemistry and geodynamic setting of the granitoids from NW Iran. Geological Journal, v. 45, pp. 451-466.

Sorkhabi, B.R. and Aritsa, K., 1997, Towards a solution for the Himalaya puzzle: mechanism of inverted metamorphism constrained by the Siwalik sedimentary record. Current Science, v. 72, pp. 862-873.

Shams, F.A., 1961, A preliminary account of the geology of the Mansehra area, District Hazara, West Pakistan. Geological Bulletin of University of the Punjab, v. 1, pp. 57-67.

Shams, F.A.,1967a, The petrology of some chloritoids and staurolitebearig schists from the Mansehra-Amb state area, northern West Pakistan. Geological Bulletin of University of the Punjab, v. 6, pp. 1-9.

Shams, F.A., 1971, The geology of the Mansehra-Amb State area, Northern West Pakistan. Geological Bulletin of University of the Punjab, v. 8, pp. 1-31.

Shand, S.J., 1943, Eruptive Rocks. John Wiley \& Sons, New York, 444 p.

Saavedra, J., 1978, Geochemical and petrological characteristics of mineralized granites of the west centre of Spain. In: Stemprok, M., Burnol, L., Tischendorf, G., (eds.), Metallization associated with acid magmatism. Geological Survey, Czechoslovakia, v. 3, pp. 279-291.

Singh, R.K.B., 2010. Geochemistry and petrogenesis of granitoids of Lesser Himalayan crystallines, Western Arunchal Himalaya. Journal of Geological Society of India, v., 75, pp. 618-631.

Singh, B., and Kumar, S., 2005, Petrogenetic appraisal of early Paleozoic granitoids of Kinnaur district, Higher Himachal Himalaya, India. Gondwana Research, v. 8, pp. 67-76.

Sarjoughian, F., Lentz, D., Kananian, A., Ao, S., and Xiao, W., 2017, Geochemical and isotopic constraints on the role of juvenile crust and magma mixing in the UDMA magmatism, Iran: evidence from mafic microgranular enclaves and cogenetic granitoids in the Zafarghand igneous complex. International Journal of Earth Sciences, v. 107, pp. $1127-1151$.

Sylvester, P.J., 1998, Post-collisional strongly peraluminous granites.
Lithos, v. 45, pp. 29-44.

Thompson, A.B., 1982, Dehydration melting of pelitic rocks and the generation of $\mathrm{H}_{2} \mathrm{O}$ undersaturated granitic liquids. American Journal of Science, v. 282 pp. 1567-1595.

Vernon, R.H., 1983, Restite, xenoliths and microgranitoid enclaves in granites. Journal and proceedings. Royal society of New South Whales, v. 116, pp. 77-109.

Villaseca, C., Orejana, D., and Paterson, B.A., 2007, Zr-LREE rich minerals in residual peraluminous granulites, another factor in the origin of low Zr-LREE granitic melts? Lithos, v. 96, pp. 375-386.

Watson, E.B., and Harrison, T.M., 1983, Zircon saturation revisited; temperature and composition effects in a variety of crustal magma types. Earth and Planetary Science Letters, v. 64, pp. 295-304.

White, A.J.R., Chappell, B.W., and Wyborn, D., 1991, Enclaves of S-type granites in the Lachlan Fold Belt, southeastern Australia. In Enclaves and Granite Petrology (J. Didier \& B. Barbarin, eds.). Elsevier, Amsterdam, v. 83 , pp. 493-507.

Waight, T.E., Mass, R., and Nicholls, I.A., 2001, Geochemical investigations of microgranitoid enclaves in the S-type Cowra granodiorite, Lachlan Fold Belt, SE Australia. Lithos, v. 56, pp. 165-186.

Wyborn, D., White, A.J.R., and Chappell, B.W., 1991, Enclaves in the Stype Cowra Granodiorite. Excursion Guide, Second Hutton Symposium on Granites and Related Rocks, Bureau of Mineral Resources, Geology \& Geophysics, Canberra, Australia, pp. 40.

Xiang, W., Xiaojuan, Y., and Chauansheng, W., 2006, Characteristic mineralogy of the Zhutishi granite: Implication for petrogenesis of the late intrusive granite. Science in China, D-Erath Sciences, v. 49, pp. 573-583.

Yurimoto, H., Duke, E.F., Peipike, J.J., and Shearer, C.K., 1990, Are discontinuous chondrite-normalized REE pattern in pegmatitic granite systems the result of monazite fractionation. Geochimica Et Cosmochimica Acta, v. 54, pp. 2141-2145.

Zen, E.A., 1988, Phase relations of peraluminous granitic rocks and their petrogenetic implications. Annual Review of Earth and Planetary Sciences v. 16, pp. 21-51.

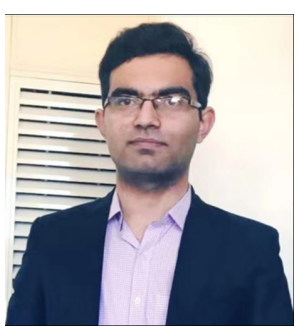

Tehseen Zafar is a $\mathrm{PhD}$ research scholar at the Institute of Geochemistry, University of Chinese Academy of Sciences. He is awardee of CAS-TWAS Scholarship and nominated in 200 researchers of the world. He is an active and dynamic researcher. His areas of interests are petrogenetic studies and geochemical evaluation of mafic-ultramafic complex. In respect of his research contribution, he has published 18 research articles including top ranked journals of Elsevier such as Lithos, Ore Geology Reviews and Geochemical Exploration. More than 10 scientific conference papers are also on his credit. 